\title{
A mesoscale study of thermal expansion behaviors of epoxy resin and carbon fiber/epoxy unidirectional composites based on periodic temperature and displacement boundary conditions
}

Kai Dong, Jiajin Zhang, Miao Cao, Minglin Wang, Bohong Gu, Baozhong Sun* College of Textiles, Key Laboratory of High Performance Fibers \&Products, Ministry of Education, Donghua University, Shanghai, China (*: Corresponding author: Email: sunbz@ dhu.edu.cn Tel: +86-21-67792782 Fax: $+86-21-67792627)$

\begin{abstract}
The thermal expansion behaviors of neat epoxy resin and carbon fiber/epoxy unidirectional (UD) composites were experimentally and numerically studied in this paper. The dynamic mechanical analysis (DMA), thermogravimetric analysis (TG), differential scanning calorimetry (DSC) and thermal conductivity measurement were used to measure the thermo-mechanical properties of epoxy resin at different temperatures. The dilatometer was used to measure the thermal strains and linear CTEs of neat epoxy resin and UD composites. In addition, a mesoscale finite element model based on the periodic temperature and displacement boundary conditions was presented to analyze the thermal expansion behaviors of UD composites. The resin-voids representative volume element (RVE) was used to calculate the thermo-mechanical properties of several kinds of resin-voids mixed matrix. From the results it can be found that the glass transition temperature of epoxy resin, porosity and fiber orientation angle have significant effects on the thermal expansion behaviors of UD composites. The mesoscale finite element analyses (FEA) have obvious advantages than various existing analysis models by comparing their predictive results. The distributions of thermal displacement, thermal stress and thermal strain were extracted between the carbon fiber, resin-voids mixed matrix and their interface, and also between the front and back surfaces of the loading direction, to further investigate thermal expansion structure effects of UD composites. This paper revealed that the mesoscale FEA based on periodic temperature and displacement boundary conditions can be also used for thermal expansion researches of other complex structure composites.
\end{abstract}

\section{Keywords}

Thermal expansion behaviors; neat epoxy resin; unidirectional (UD) composites; mesoscale finite 
element analyses (FEA) 


\section{Introduction}

Carbon fiber-reinforced composites are currently more and more used in structural applications that involve various temperature ranges [1]. When excessive temperature differences occur in a composite structure or its surrounding, the disequilibrium of the potential volumetric changes or mismatch of structural deformations in this kind of composite are very complicated. A large rise in device temperature can lead to the fracture, delamination, melting, creep, electro-migration and even burning of packing materials [2]. The resistance capacity to these thermal failure modes of composites is always attributed to their thermal properties, such as thermal conductivity, specific heat and coefficient of thermal expansion (CTE). And CTE is an imperative property to define the end use application that should be firstly considered in these occasions. An understanding and ability to predict the CTE in composites is an important design criterion for producing dimensionally stable structures, especially if they are assemblages of many different types of materials [3]. Recently, many novel experimental methods have been developed to measure the CTE of polymeric materials, such as electronic speckle pattern interferometry [4], digital image correlation method [5], fiber bragg grafting sensor [6], infrared thermography [7], atomic force microscopy [8] and Raman spectroscopy [9], etc. Although they each have advantages in the measurement of CTE, all of them need sophisticated equipments, longer measuring time and more number of repeated trials and will also lead to larger error variations. Furthermore, the internal structure effects of thermal expansion, thermal-mechanical coupling relationship and thermal failure mechanisms of composites cannot be revealed from them. Therefore, a more convenient and more comprehensive analytical model is urgently needed to characterize thermal expansion behaviors of composites in a wide range of temperatures.

Considering the internal structure effects of UD composites on the thermal expansion, the thermal expansion properties of its constituents should be firstly determined and analyzed. It is well known that carbon fiber-reinforced composites are manufactured by carbon fiber reinforced body impregnated with resin matrix. And during their processing, there will also be some voids existing in composites. Therefore, to a great extent, the CTEs of these composites depend on the CTEs of its constituents. From this perspective, some researchers studied the CTEs of carbon fibers and epoxy resin. For example, Sauder et al. [10], Rupnowski et al. [11] and Pradere et al. [12, 13] studied the CTEs of carbon fiber in a wide temperature range, which showed that the effective 
longitudinal and transverse CTEs of carbon fibers almost maintained stable during the temperatures from room temperature to $300{ }^{\circ} \mathrm{C}$. However, the fiber orientation angle $[14,15]$ may have a remarkable effect on the thermal expansion behaviors of UD composites. Due to the high temperature sensitivity of epoxy resin, its CTE may have a significant effect on the final thermal expansion behaviors of carbon fiber/epoxy composites. For example, Glavchev et al. [16], Tognana et al. [17] measured and analyzed the CTEs of pure epoxy resin matrix as well as its compounds. Both of their research found that the glass transition temperature $T_{g}$ had a great influence on the CTE of epoxy resin and the CTE of epoxy resin had an important role in the final CTEs of composite materials. Although the porosity of voids [18] in composites is very little, its effect on thermal expansion is very noteworthy. Therefore, effective determination the CTEs of epoxy resin, investigation the effects of fiber orientation and porosity are also important parts to accurately analyze the thermal expansion behaviors of composite materials.

For the numerical analytical models about the thermal expansion of composites, many researchers conducted a series of works. At the beginning, various kinds of micromechanical analytical models have been presented to predict the CTEs of UD composites, such as rule of mixture (ROM), Turner [19, 20], Kerner [20, 21], Levin [22], Schapery [23], Van Fo Fy [24, 25], Chamis [26], Chamberlain [27], Geier [28], Schneider [27], Rosen \& Hashin [29] and so on. However, due to many idealistic assumptions, these analytical models may have more or less shortcomings in terms of the CTE predictive ability. In addition, the structure effects and interfacial effects cannot be illustrated from these models. In this paper for facilitating comparisons, the detailed analyses about these micromechanical models are also made in Appendix A. Recently, with the improvement of computation capability of computer, many complicated numerical solutions, including perturbation-based stochastic homogenization analysis method [30], effective thermoelastic approach [31], Mori-Tanaka (M-T) method [32] and the finite element method (FEM) [33-38], are more and more used to predict the thermal expansion behaviors of composites. Among these, the finite element method is the most widely used for the CTEs prediction of carbon fiber-reinforced composites. For example, Karadeniz et al. [33] used the FEM to study the effective CTEs of fiber reinforced composites by micromechanical modeling. Their results showed that the FEM and existing analysis models were in good agreement with experimental for 
longitudinal CTEs, while for transverse CTE the FEM results showed better agreement with experimental data. Similar research was also done by Ran et al. [34], who not only analyzed the longitudinal and transverse CTEs of UD composite, but also indicated that the CTEs were mostly affected by the fiber to matrix stiffness ratio and the fiber to matrix CTE ratio. Dong [35] developed a regression-based model combined with FEM for predicting the transverse CTEs of UD composites. The result showed that the developed model offered excellent accuracy while reduced complicated computation process. Xu et al. [36] developed a strain energy model based on the relationship established between strain energy of the microstructure and that of homogenized equivalent model under specific thermo-elastic boundary conditions to predict the effective CTEs of composite materials. Accordingly, Abueidda et al. [37] investigated the effective CTE of novel interpenetrating phase composites by generating several three-dimensional unit cells models. Wang et al. [38] investigated the distribution and development of the thermal-shock-induced stress in ceramic-matrix composites by FEA. They found that the thermal stress failure always occurred at or near the heat part of the fibers. Although the prediction of FEM showed great advantages over other analytical models, most of them didn't consider the effect of temperature or ignored the temperature dependence of thermal and mechanical properties of carbon fibers and epoxy resin. In fact, the elastic modulus, thermal conductivity, specific heat capacity and CTEs of carbon fibers and epoxy resin are all temperature dependent. Therefore, both the thermal and mechanical properties of materials at different temperatures must be incorporated in finite element analysis for accurately predicting the CTEs of UD composites. Although some studies [39-42] may have already taken the temperature effects into account during the thermal expansion analysis of composites, the thermal-mechanical coupling effects on CTEs of composites have never been revealed. The thermal stress induced by the temperature variations, due to the CTEs mismatch between the fiber and the matrix, can greatly affect the mechanical properties of composite. On the contrary, the change of mechanical properties may also lead to the change of temperatures. Therefore, a more comprehensive thermal-mechanical coupling finite element analysis should be conducted.

Based on the above unsolved problems, we constructed a mesoscale finite element model in the present work to analyze the thermal expansion behaviors of epoxy resin as well as carbon fiber/epoxy UD composites at the temperature range of $20-300^{\circ} \mathrm{C}$. The thermal-mechanical 
coupling relation is realized by applying the periodic temperature and displacement boundary conditions to the mesoscale RVE. This study helps for further understanding the thermal expansion behaviors of epoxy resin and UD composites, but it can also provide an efficient method for thermal expansion research of complex structure composites. The paper is structured as follows. In section 2, the involved materials and experimental methods for temperature-dependent thermomechanical of neat epoxy resin and UD composites were presented. In section 3, the detailed finite element analyses were made, including the mesoscale microstructure model, the computing method of thermal strain/stress, the periodical boundary condition and the meshing scheme. The detailed experimental and FEA results as well as their comparisons are given and discussed in section 4. In section 5, a few concluding marks are provided.

\section{Experimental}

\subsection{Materials and sample preparation}

An epoxy resin JC-02A based on diglycidyl ether of bisphenol A (Changshu Jaffa Chemical Co., Ltd) with hardener JC-02B (improved methyl tetrahydrophthalic anhydride) and accelerant JC-02C (tertiary amine) was used as matrix material. The mixed proportion of resin, hardener, and accelerant was 100:80:0.5 by weight. The uncured epoxy resin was first vacuumed to remove the air bubbles before being used to make composites. The neat epoxy resin was cured by directly injecting them into rectangular mold. The curing temperature for neat resin was $90^{\circ} \mathrm{C}$ for $2 \mathrm{~h}, 110^{\circ} \mathrm{C}$ for $1 \mathrm{~h}$ and finally $130^{\circ} \mathrm{C}$ for $4 \mathrm{~h}$. The unidirectional carbon fiber pre-impregnated (prepreg) that was made up of carbon fibers (T700S-12K) and the same epoxy resin as above, was used to prepare the UD composites with the help of assisted vacuum pump pressure. The UD composite material with the thickness about $6 \mathrm{~mm}$ was laid up by 42 layers carbon fiber prepregs along the same direction. The curing temperature for UD composites was $130^{\circ} \mathrm{C}$ for $2 \mathrm{~h}$. The final specimens were removed from the oven after cooling for $12 \mathrm{~h}$, and then cut into the required sizes and directions $\left(0^{\circ}, 90^{\circ}\right.$ and $\left.45^{\circ}\right)$ by means of high-pressure water jet. Each test needs at least three samples and each sample will be repeated at least two times. The average fiber packing fraction of UD composites was $0.675_{-0.078}^{+0.12} \%$ (the superscript and subscript were the maximum allowed error values) measured from ten UD 
composite samples by the combustion method in muffle furnace. The test temperature was set as $600^{\circ} \mathrm{C}$ and the burning time was $6 \mathrm{~h}$ to thoroughly remove the resin. Before the thermal expansion tests, all specimens were dried in oven at $60^{\circ} \mathrm{C}$ for $1 \mathrm{~h}$ to eliminate the effects of moisture and then natural cooled to room temperature. All the tests were conducted in standard conditions at a room temperature of $25 \pm 2^{\circ} \mathrm{C}$ and $65 \pm 2 \%$ relative humidity to ensure its reliability.

\subsection{Dynamic mechanical analysis (DMA)}

The mechanical behaviors of the epoxy resin at different temperatures were tested with dynamic mechanical analysis (DMA, Perkin-Elmer DMA-8000). The temperature of the sample or the frequency of the stress is often varied, leading to variations in the complex modulus. This approach can be used to locate the glass transition temperature, as well as to identity transitions corresponding to other molecular motions [43]. In order to understand the mechanical behaviors of the epoxy resin under high temperature, temperature sweep DMA test of the resin was conducted by single cantilever bending model at the ramp rate $3^{\circ} \mathrm{C} / \mathrm{min}$. The temperature sweep range was from room temperature to $220{ }^{\circ} \mathrm{C}$, and the size of resin specimen was $15.8 \times 5.5 \times 2.8 \mathrm{~mm}$.

\subsection{Thermogravimetric analysis (TG)}

The onset temperature of thermal degradation of epoxy resin was determined by the thermogravimetric analysis (NETZSCH TG 209 F1). The TG analysis was conducted under a nitrogen atmosphere in the temperature range between $20^{\circ} \mathrm{C}$ and $600^{\circ} \mathrm{C}$. The mass loss and derivative thermogravimetric (DTG) curves were obtained at a heating rate of $10^{\circ} \mathrm{C} / \mathrm{min}$. The onset temperature of degradation was determined by the intersection of the tangents of the peak with the extrapolated baseline from the first degradation peak of the curves [44].

\subsection{Measurement of thermal conductivity}

The thermal conductivities of the neat epoxy resin and UD composites were conducted by the thermal conductivity testing instrument DZDR-S based on the transient plane source (TPS) technique [45] as well as the ASTM C 177-97. The testing specimens and instrument are shown in

Fig. 1. The sensor, typically double concentric ring with multiple spirals of nickel wire, is sealed between two Kapton films for electrical insulation. The constant current is passed through the 
sensor at the beginning of the experiment. The temperature increase in sensor is monitored by resistance charge. The thermal property of the sensor at various temperatures is a known quantity. The conductivity of a sample can then be obtained by solving the differential equation of conduction [46].

\subsection{Measurement of specific heat}

The determination of specific heat at constant pressure, $C_{p}$, was performed in according to the ASTM E 1269 by means of differential scanning calorimeter technique (DSC, NETZSCH 214). A synthetic sapphire disk ( $\alpha$-aluminum oxide) was used as the standard reference material. The DSC measurements of 5.6-5.9 mg of the neat epoxy resin were carried out in opened alumina crucibles under a nitrogen atmosphere in a temperature range from 25 to $300{ }^{\circ} \mathrm{C}$ with a heating rate of $10^{\circ} \mathrm{C} / \mathrm{min}$.

\subsection{Measurement of coefficients of thermal expansion}

The coefficients of thermal expansion measurements of the neat epoxy resin and UD composites were conducted with a classical dilatometer (ZRPY-1000). It consists of an oven, an alumina sample support, a quartz glass pressing bar, thermocouples, and LVDT (linear various differential transformers) sensors. The test samples and the dilatometer are shown in Fig. 2. The sample placed on an alumina support was held with a pressing bar. The length variations during the heating are recorded and sent to the acquisition system. The output data contains time, elongation of the sample, and temperature. The detailed role of each component of the dilatometer is described in reference [47].

The instantaneous CTE $\alpha$ was evaluated based on the following equation that is defined as the slope of the relative length change at the respective temperature:

$\alpha=\frac{\Delta L-K}{L_{o}\left(T_{2}-T_{1}\right)}$

where $\Delta L$ is the linear length change when the sample was heated to from temperature $T_{1}$ to temperature $T_{2}$, And $\Delta L=L-L_{o}$, where $L$ and $L_{o}$ are the sample length at temperature $T_{2}$ and 
temperature $T_{1}$, respectively. In order to eliminate the thermal expansion effects of the pressing bar and the sample holder, the system compensation value was used. The system compensation value $K$ at temperature $T_{2}$ can be obtained from the reference quartz sample, and can be calculated from the following equation:

$$
K=\Delta L_{r e f}-\alpha_{r e f} L_{r e f}\left(T_{2}-T_{1}\right)
$$

where $\Delta L_{r e f}$ is the linear length change of the reference quartz sample at temperature $T_{2} . L_{r e f}$ is the length of reference quartz sample at temperature $T_{1}$ and $\alpha_{\text {ref }}$ is the coefficient of thermal expansion of the reference quartz sample. Fig. 3 shows the experimental calibration thermal displacement and CTE curves of the reference quartz glass sample, which is in accordance with the theoretical value $0.55 \times 10^{-6} /{ }^{\circ} \mathrm{C}$.

\section{Finite element analyses}

In this paper, the finite element analyses were operated on commercial available finite element software package ABAQUS/Standard (ver. 6.12) under the environment of operating system LINUX. The mesoscale finite element model was directly established in ABAQUS and the coupled temp-displacement analysis step was created to analyze the thermal expansion behaviors. The powerful meshing software HyperMesh (ver. 12.0) was used to generate the meshes of the mesoscale model for making the fibers and resin share the same nodes at their interface. The periodic displacement and temperature boundary conditions were imposed on the nodes of the mesh in the mesoscale model. The computing methods of thermal strain and thermal stress in FEA were described.

\subsection{Mesoscale model}

In the UD composites, only three components are existed: fibers, resin and a few voids. The microstructure observations of UD composites shown in Fig. 4 by SEM (scanning electron microscopic, TM-3000, Japan) indicates that the resin is filled in the spaces between carbon fibers, and the voids are mainly stochastic distributed at the interface between fibers and resin. Considering the UD composites include hundreds of fibers impregnated in the matrix with 
randomly packed pattern in the transverse direction, a hexagonal array distribution of fibers in UD composite was assumed. For the fiber packing patterns in resin, rectangular cross-section, column model and hexagonal model have been investigated. Among them, the hexagonal model is widely applied for modeling transversely properties of the matrix impregnated fiber yarns [48]. Considering the distribution of voids is stochastic and complex, the resin-voids mixed model with the dimensions of $1 \times 1 \times 1 \mathrm{~mm}$ is used base on the equivalent volume method, i.e. the RVE of voids whose radius is $0.06 \mathrm{~mm}$, is regularly distributed on the four corners of the mixed model. In order to investigate the thermal expansion behaviors of UD composites, a mesoscale analysis technology is adopted with application of the periodic boundary conditions. A mesoscale RVE as shown in Fig. 5 can be introduced to analyze the thermal expansion behaviors of UD composites. Considering the convergence and calculating time, the dimension of the mesoscale RVE is set as $1 \times 1.8 \times 0.5 \mathrm{~mm}$. The diameter of the circular-section fiber is $0.88 \mathrm{~mm}$ based on the fiber packing fraction in UD composites. The porosity of voids was assumed as average area ratio calculated from lots of SEM photographs, e.g. two photos in Fig. 4, which were mainly selected from the cross-sections of UD composites along their thickness directions. The average area ratio calculated from multiple cross-sections was assumed equal to the volume ratio. After making a statistical analysis about these results, the porosity of UD composites was approximately equal to $1.2 \%$. In this paper, the voids were regarded as solid air and the carbon fiber was assumed as transverse isotropic material. Except the specific heat and thermal conductivity of carbon fiber, the other thermal mechanical properties of carbon fibers and those of voids were all assumed as constant, which were listed in Table 1.

In this paper, a progressive finite element analysis method was used to analyze the thermal expansion properties of UD composites. The resin-voids RVE was first used to calculate the thermal and mechanical properties of the resin containing voids, and then the thermal and mechanical properties of the resin containing voids (resin-voids mixed matrix) and carbon fibers were used to analyze the thermal expansion behaviors of UD composites.

\subsection{Calculation of thermal strain and thermal stress}

In ABAQUS, if the CTE is a function of temperature, the value of the reference temperature is 
needed. Therefore, they generated thermal strain matrix $\varepsilon_{t h}$ according to the formula

$$
\varepsilon_{t h}=\alpha(T)\left(T-T^{0}\right)-\alpha\left(T^{I}\right)\left(T^{I}-T^{0}\right)
$$

where $T, T^{I}$ and $T^{0}$ are the current temperature, the initial temperature and the reference temperature respectively. $\alpha(T)$ and $\alpha\left(T^{I}\right)$ are the CTE matrix of the current temperature and reference temperature respectively. The second term in Eq. (3) represents the strain due to the difference between the initial temperature and the reference temperature. This term is necessary to enforce the assumption that there is no initial thermal strain for cases in which the reference temperature does not equal the initial temperature.

When a structure is not free to expand, a change in temperature will cause stress and constrained thermal expansion can cause significant stress. Accordingly, the thermal stress matrix $\sigma_{t h}$ can be calculated as

$$
\sigma_{t h}=-\alpha(T) C(T)\left(T-T^{0}\right)
$$

where $C(T)$ is the stiffness matrix at temperature $T$.

\subsection{Periodic boundary conditions}

There are several boundary conditions used for the analysis and prediction of the overall behaviors of composites. However, many researchers $[49,50]$ have indicated that the 'plain-remain-plain' boundary conditions are over-constrained boundary conditions. Actually, these boundary conditions are not only over-constrained but may also violate the stress/strain periodicity conditions. The periodic boundary conditions (PBCs) developed by Xia et al. [51, 52] provide an explicit unified form application in finite element analysis of unit-cells for UD composites. Here, the procedures to implement the PBCs to the RVE both for displacement and temperature fields in finite element analysis by master-slave node technique are described.

For a 3D periodic structure, the displacement field can be expressed by

$u_{i}\left(x_{1}, x_{2}, x_{3}\right)=\bar{\varepsilon}_{i k} x_{k}+u_{i}^{*}\left(x_{1}, x_{2}, x_{3}\right)(i, k=1,2,3)$

where $\bar{\varepsilon}_{i k}$ are the global (average) strains applied to the periodic body and $x_{k}$ is the coordinate of

a material point in Cartesian system. In the above, $\bar{\varepsilon}_{i k} x_{k}$ represents a linear distributed 
displacement field and $u_{i}^{*}\left(x_{1}, x_{2}, x_{3}\right)$ is the periodic part of the displacement which is repeated from one unit-cell to another.

This displacements on a pair of parallel opposite boundary surfaces denoted as ' $j+$ ' and ' $j$-' are expressed as

$u_{i}^{j+}=\bar{\varepsilon}_{i k} x_{k}^{j+}+u_{i}^{*}$

$u_{i}^{j-}=\bar{\varepsilon}_{i k} x_{k}^{j-}+u_{i}^{*}$

For any periodic structure, the $u_{i}^{*}\left(x_{1}, x_{2}, x_{3}\right)$ is the same at two parallel boundaries. Therefore, the difference between the above-mentioned two equations can be rewritten as $u_{i}^{j+}-u_{i}^{j-}=\bar{\varepsilon}_{i k}\left(x_{k}^{j+}-x_{k}^{j-}\right)=\bar{\varepsilon}_{i k} \Delta x_{k}^{j}$

where $\Delta x_{k}^{j}$ is a constant for any pair of corresponding points on the two parallel boundary surfaces. And the right side of the Eq. (8) becomes constant with specified global strains $\overline{\mathcal{E}}_{i k}$. As a special type of displacement boundary conditions, Eq. (8) can be easily applied in the finite element analysis as nodal displacement constraint equations. The application of Eq. (8) can guarantee satisfaction of the continuity of displacement and traction at the opposite parallel boundary surfaces as proved by Kuo [53].

The Eq. (8) is applied by master and slave nodes technique as illustrated in Fig. 6. In the unit-cell with periodicity in all three directions, $\xi-\xi^{\prime}, \eta-\eta^{\prime}$ and $\zeta-\zeta^{\prime}$ are pairs of periodic nodes locating on the opposite parallel boundary surfaces of the unit cell and the lines linking them are parallel to the three coordinate axes, respectively. The rigid body translations and rotations of the unit cell can be constraint by setting point $A$ to be fixed, point $B$ to be constraint in $x_{2}$ direction and point $D$ to be constraint in $x_{1}$ and $x_{2}$ directions. Taking the corner points $A^{\prime}, B$ and $D$ as the master nodes, the Eq. (8) can be reduced as

$u_{i}^{\xi}=u_{i}^{\xi^{\prime}}+u_{i}^{B}, u_{i}^{\eta}=u_{i}^{\eta^{\prime}}+u_{i}^{A^{\prime}}, u_{i}^{\zeta}=u_{i}^{\zeta^{\prime}}+u_{i}^{D}$

In the finite element model, the meshes and nodes on the two opposite surfaces of the unit-cell should be same for applying the above Eq. (9). The implementation of Eq. (9) can be further divided into three categories for the corner nodes, the nodes on the edges and the rest nodes on the 
boundary surfaces.

For the corner nodes $C, D^{\prime}, B^{\prime}$ and $C^{\prime}$, Eq. (9) is reduced to

$u_{i}^{C}-u_{i}^{D}=u_{i}^{B}, \quad u_{i}^{D}-u_{i}^{D^{\prime}}=u_{i}^{A^{\prime}}, u_{i}^{B^{\prime}}-u_{i}^{A^{\prime}}=u_{i}^{B}, u_{i}^{C^{\prime}}-u_{i}^{C}=u_{i}^{A^{\prime}}$

For the nodes on the edges of the unit cell except the corner nodes, Eq. (9) would be expressed by Eq. (11).

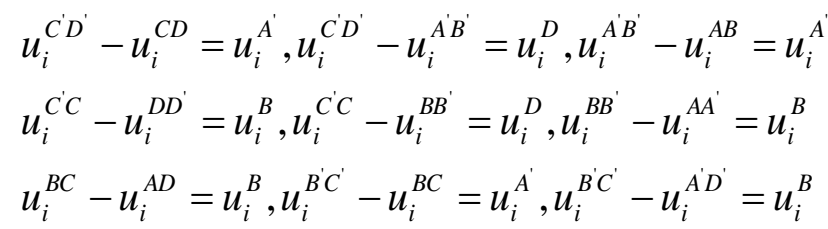

In Eqs. (9)-(11), $i(=1,2,3)$ represents the Cartesian coordinate direction. For all the rest nodes on boundary surfaces, Eq. (9) is applied directly.

Based on the periodic displacement boundary conditions described above, Dong et al. [54] developed the periodic temperature boundary conditions, also according to the master and slave nodes technique. The equations for establishing temperature PBCs are summarized in the following:

- Surfaces

$T^{\xi^{\prime}}-T^{\xi}=T^{D}, T^{\eta^{\prime}}-T^{\eta}=T^{B}, T^{\zeta^{\prime}}-T^{\zeta}=T^{A^{\prime}}$

- Edges

$T^{C^{\prime} D^{\prime}}-T^{C D}=T^{A^{\prime}}, T^{C^{\prime} D^{\prime}}-T^{A^{\prime} B^{\prime}}=T^{D}, T^{A^{\prime} B^{\prime}}-T^{A B}=T^{A^{\prime}}$
$T^{C^{\prime} C}-T^{D D^{\prime}}=T^{B}, T^{C^{\prime} C^{\prime}}-T^{B B^{\prime}}=T^{D}, T^{B B^{\prime}}-T^{A A^{\prime}}=T^{B}$
$T^{B C}-T^{A D}=T^{B}, T^{B^{\prime} C^{\prime}}-T^{B C}=T^{A^{\prime}}, T^{B^{\prime} C^{\prime}}-T^{A^{\prime} D^{\prime}}=T^{B}$

- Corners

$T^{C}-T^{D}=T^{B}, T^{D}-T^{D^{\prime}}=T^{A^{\prime}}, T^{B^{\prime}}-T^{A^{\prime}}=T^{B}, T^{C^{\prime}}-T^{C}=T^{A^{\prime}}$

It should also be noted that the temperature loadings and boundary condition applications should be dependent on corresponding degree of freedom. In the ABAQUS user manual [55], the displacement degrees of freedom of the three orthogonal directions are 1,2 and 3 respectively and the temperature degree of freedom is 11.

\subsection{Meshing}


The implement of PBCs needs the opposite parallel boundary surfaces having the same mesh configurations and node distributions. In addition, the meshes of matrix should share the same nodes with those of carbon fiber at their interface in order to obtain a stable transferring of thermal and mechanical loadings. Considering these requirements, the HyperMesh software was used. The surface quadrilateral meshes were firstly generated, and then these meshes were solid mapped by line dragging to generate the hexahedral meshes. The mesh sensitivity analysis has also been done and the final element size of the mesoscale RVE was chosen as 0.15 as a result of reducing computational cost as well as ensuring the computation quality. The number of nodes for the three primary axes $(x, y, z)$ was 65,119 and 4 , respectively. Accordingly, the number of nodes for the three boundary surfaces $(x y, y z, x z)$ except the nodes on the edges was 8612, 476 and 260, respectively.

\section{Results and discussions}

\subsection{Thermal and mechanical properties of epoxy resin}

\subsubsection{DMA and TG}

As shown in Fig. 7, the temperature sweep DMA test of epoxy resin found that the storage modulus decreases sharply from 105 to $115{ }^{\circ} \mathrm{C}$, which indicates that $105-115{ }^{\circ} \mathrm{C}$ is the glass transition region and that $110{ }^{\circ} \mathrm{C}$ is the $T_{g}$ of the epoxy resin. The mechanical properties of epoxy resin at any temperature point can be obtained from the DMA curves. As shown in Fig. 8, the TG test of epoxy resin indicated that the onset temperature of thermal degradation was around $300{ }^{\circ} \mathrm{C}$ and the temperature of complete thermal degradation was close to $600{ }^{\circ} \mathrm{C}$. Because the chemical compositions of epoxy resin change greatly after thermal degradation [56], therefore, it makes no sense to study the thermal expansion properties of composites after the temperature of thermal degradation. According to the DMA and TG curves of epoxy resin, the thermal mechanical properties of epoxy resin during the glass transition temperatures experienced tremendous change. Therefore, the selected nine temperature points, i.e. $35,80,90,110,120,130,150,180$ and $210^{\circ} \mathrm{C}$, were mainly distributed around the temperature $T_{g}$ to explore the thermal expansion behaviors of UD composites in the finite element analyses. 


\subsubsection{Specific heat capacity and thermal conductivity}

Typical three heat flux curves for cured epoxy resin obtained from DSC measurement are shown in Fig. 9. A good consistency can be found from them and the glass transition of cured epoxy resin was detected around $110{ }^{\circ} \mathrm{C}$, i.e. the transition from the amorphous-solid state to a viscous-fluid phase. The small standard deviations in the measurements may be due to the fact the specimens used in the testing are very small. As a result they cannot adequately represent the macroscopic property of the composites due to its inhomogeneity. Therefore, the average values of DSC curves combining with those of reference sapphire specimens were used to calculate the specific heat capacity of cured epoxy resin at different temperatures. In this study the measured heat capacity for neat cured epoxy resin at room temperature is $1248 \mathrm{~J} \mathrm{~kg}^{-1} \mathrm{~K}^{-1}$ which is consistent to previously reported values, $1200 \mathrm{~J} \mathrm{~kg}^{-1} \mathrm{~K}^{-1}$ in references [54, 57].

The thermal conductivity of neat cured epoxy resin at room temperature was measured by Hot Disk technique, which was $0.1843 \mathrm{~W} \mathrm{~m}^{-1} \mathrm{~K}^{-1}$. While for its values at elevated temperatures, our previous paper [57] has already made the detailed works.

\subsubsection{Coefficient of thermal expansion}

The experimental thermal strain and linear CTE of neat cured epoxy resin as a function of temperature are shown in Fig. 10. Both the thermal strain and linear CTE curves of epoxy resin could be divided into three regions, i.e. the glassy region (I and II), the glass transition region (III) and the rubbery region (IV). The thermal strain curve showed two approximate linear thermal strain rising processes with the increase of loading temperatures. The first approximate linear process corresponded to the glassy region and the second process was fallen into the rubbery region. Before the $T_{g}$, thermal strain curve showed an approximate linear increasing trend with the linear slope of 3.57. While after the $T_{g}$, the thermal strain curve showed another approximate linear rising with a little higher linear slope of 8.71. In glassy region, the molecular chains or the chain segments can't move and only the atoms or groups of molecules vibrate in their equilibrium positions. Therefore, the thermal strain increased very slowly as temperature rising. However, the thermal strain increased faster in the rubbery region due to the molecular chain segment movement. Around the glass transformation temperature there is obvious shrinkage of the thermal 
strain curve during the heating process, which can be attributed to the molecular chain contraction of amorphous region.

The slope of the thermal strain versus temperature curve gives the CTE results. Although the thermal strain versus temperature curve was approximate linear in glassy and rubbery regions, there was still difference between the adjacent temperature points which resulted in the instantaneous CTEs of epoxy resin were not constant at each individual region. Just as the partial enlarge figure in Fig. 10, the actual thermal strain curve had a slight shrinkage at temperature $60^{\circ} \mathrm{C}$, which was also the reason why there was a slight shrinkage in the glassy region of CTE curve. At the beginning of heating process, the post curing as well as chemical cross-linking of the cured epoxy resin would make the CTE curve first decrease. In addition, the relaxation of the residual stress-strain existing in the epoxy resin during the curing process could also lead to this phenomenon. After the short declining process, the CTE curve will begin to rise due to the physical and chemical transformations. It is important to point out that the thermal expansion of epoxy resin is usually associated with the molecular chains dilatation and the free volume dilatation. The main contribution to CTE in the glassy region could be attributed to chains dilatation. Conversely, in the rubbery region the free volume dilatation could be the main mechanism [17]. Because the role of the molecular chains dilatation at low temperatures is very limited, the stage II shows a very little increase. On the contrary, the function of free volume dilatation to thermal expansion at high temperature is very prominent. Just as the stage IV, the linear CTE curve presents a larger linear increase in the rubbery region. The glass transition region, (i.e. stage III) different from the glassy region and rubbery region, shows a significant decline of the linear CTE. The obvious shrinkage of the linear CTE curve around the glass transformation temperature could also be attributed to the contractions of crystalline lattices.

\subsection{Resin-voids RVE}

The resin-voids RVE was used to obtain the thermal and mechanical properties of epoxy resin containing voids at different temperature points. In order to further reflect the effect of porosity on the thermal expansion, extra three kinds of resin-voids RVEs with different porosities, i.e. matrix with no voids, with $5 \%$ voids and with $10 \%$ voids, were chosen to compare their thermal expansion behaviors with the actual model, whose porosity was $1.2 \%$. Because the epoxy resin 
and voids are both isotropic materials, the resin-voids RVE can also be regarded as an isotropic material. After applying periodic displacement and temperature boundary conditions to the RVEs and also the temperature loadings to point $\mathrm{D}$, the thermal expansion properties of these resin-voids RVEs can be easily obtained. The selected nine temperature points were applied to the four resin-voids RVEs, respectively. The obtained engineering thermal strains and linear CTEs as a function of temperature of these resin-voids RVEs are shown in Figs. 11 and 12, respectively. And accordingly, the corresponding distributions of the thermal mechanical behaviors of these resin-voids RVEs are illustrated in Fig. 13. The FEA results with no voids were significantly closer to the experimental. And it was obvious that the thermal strain and CTE decreased with the increase of porosity. Because voids existed in composites could not only provide more space for the free volume expansion, but could also lower the elastic modulus of composite, therefore, they could effectively reduce the CTE of cured epoxy resin. However, both the experimental thermal strain and linear CTE results during the glass transition region were lower than those of FEA, which could be attributed to the relaxation of the residual stress-strain existing in the samples around the glass transition temperature. The FEA didn't take the stress relaxation factor into consideration.

\subsection{CTEs of UD fiber reinforced composites}

\subsubsection{Effect of porosity}

In this paper, it was assumed that the voids only affect the thermal mechanical properties of epoxy resin matrix. Therefore, according to the thermal mechanical properties of above resin-voids RVEs combing with the basic thermal mechanical properties of carbon fiber, the linear CTEs of UD fiber reinforced composites could be effectively calculated by FEA. Fig. 14 shows the linear CTEs of UD fiber reinforced composites with different porosities. The obvious effect of porosity on the thermal expansion behavior of UD composites is found from these curves. The higher the porosity is, the lower the linear CTE will be. The changing regularities of these curves are the same, which means that the voids only change the extent of thermal deformation rather than the state. The entrained air voids offer more room for the expansion and accommodate a part of the internal thermal expansion of solid skeleton. In addition, the voids existing in epoxy resin can also reduce the elastic modulus of resin-voids RVEs. Therefore, the voids can effectively reduce the CTE of 
UD fiber reinforced composites.

\subsubsection{Effect of fiber orientation angle}

In addition to the longitudinal $\left(0^{\circ}\right)$ and transverse $\left(90^{\circ}\right)$ linear CTEs of UD composites, the linear CTE of $45^{\circ}$ direction was also measured in order to characterize the effect of fiber orientation angle on the thermal expansion behaviors of UD composite. Fig. 15 shows the experimental thermal strain and linear CTE curves of UD composites with different fiber orientation angles. It was found that the thermal strains and linear CTEs increased with the increase of fiber orientation angle. This is due to the anisotropic characteristics of carbon fiber determining its axial CTE lower than its radial CTE. The longitudinal linear CTEs of UD composites are mainly dependent on the axial CTE of carbon fiber, while its transverse linear CTEs are mainly dependent on the CTE of epoxy resin. In addition, elastic modulus in a certain direction can be used as an indicator of hindrance of fibers to thermal expansion in that direction. Higher is the elastic modulus, lower the CTE will be. Elastic modulus of UD composites is highest along fiber axial direction and lowest along fiber radial direction. Therefore, the CTE of UD composites along $0^{\circ}$ lay-up direction should be minimum, while have a maximum value along $90^{\circ}$ lay-up direction.

\subsubsection{Comparison of results with different methods}

In this paper, in order to demonstrate the capability of FEA in prediction the thermal expansion properties of UD composites, several existing analytical models were also used to compare their predicting results with FEA. Fig. 16 shows the comparative results of UD composites between experimental, FEA and various analysis models. It turned out the mesoscale FEA could better predict the thermal expansion behaviors of UD composites than the various analytical models, and the Rosen and Hashin model shows closer to the experiment and FEA comparing with other analytical models. Although these various analytical models couldn't accurately predict the linear CTEs of UD composites, they presented the same changing trends with the experimental and FEA, which showed the feasibility of the CTE calculation for UD composites by these analysis models. Due to the negative axial CTE of carbon fiber, the longitudinal linear CTE of UD composites also showed negative after the glass transition temperature. The transverse linear CTE of UD composites showed the same changing trends as the neat cured epoxy resin, but its values were 
significantly less than those of neat cured epoxy resin. The reason could be explained by that the fiber radial CTE was lower than that of epoxy resin and the added carbon fibers could effectively reduce the CTE of UD composites.

Figs. 17 and 18 show the distribution maps of the thermal mechanical behaviors, i.e. thermal stress, thermal strain, thermal displacement, temperature and heat flux density of UD composites, as a function of temperature in the longitudinal and transverse directions respectively. Obvious temperature-dependent can be found from these pictures, which show increase with the increment of temperature. However, in the transverse direction, the maximal thermal stress appears during the glass transition regions. This phenomenon can be attributed to that the epoxy resin master the main transverse properties of UD composites, which have thermal stress concentration due to molecular chain contraction at glass transition temperature. For the longitudinal direction, the maximal thermal displacement occurs during the glass transition regions. The glass transition temperature of epoxy resin and the negative axial CTE of carbon fiber could lead to this.

\subsection{Distributions of thermal displacement, thermal stress and thermal strain}

\subsubsection{Between fiber, resin-voids mixed matrix and their interface}

In order to characterize the internal thermal expansion effects of UD fiber reinforced composites, the thermal displacement, thermal stress and thermal strain of the loading direction were extracted from the carbon fiber, resin-voids mixed matrix and their interface, respectively. Just as shown in Fig. 19, three nodes, i.e. 52930, 52929 and 52942 representing the carbon fiber, resin-voids mixed matrix and their interface, respectively, were extracted at different temperature points. Both the longitudinal $(z z)$ and transverse $(x x)$ loadings indicated that the thermal stresses in the carbon fiber were larger than those in the matrix, and in contrast, the thermal displacements and thermal strains in the matrix were larger than those in the carbon fiber. The thermal strains showed the same changing trends as the thermal displacements and the thermal strains in the carbon fiber were close to zero, which were much lower than those in the matrix. These phenomena could be attributed to the different elastic modulus and CTE between carbon fiber and epoxy resin. The higher elastic modulus and lower linear CTE of carbon fiber determined its higher thermal stress and lower thermal strain, while the lower elastic modulus and higher linear CTE of epoxy resin determined the lower thermal stress and higher thermal strain of the mixed matrix. In addition, the 
glass transition temperature has an obvious effect on the thermal displacements, thermal stresses and thermal strains of the mixed matrix, while it has a neglected effect on the carbon fiber, which means that the effect of the glass transition temperature on the thermal expansion behaviors of UD composites is mainly dependent on the epoxy resin.

There are also some differences between longitudinal and transverse loadings. For example, in the longitudinal loadings the thermal stresses of carbon fiber were negative and the interface showed the larger thermal stress values comparing with carbon fiber and matrix. However, in the transverse loadings the thermal stresses of carbon fiber were positive and the interface showed the lower thermal stress values than the carbon fiber and matrix. The negative thermal stresses were provided by the compressive loadings and the positive thermal stresses were provided the tensile loadings. Therefore, in the longitudinal direction the carbon fiber was suffered from compressive force, while it was suffered from the tensile force in the transverse direction. In addition, the directional inconsistent CTEs between the carbon fiber and matrix led to the thermal stress easier to concentrate at their interface. While the interface thermal stress could be reduced if the directions of CTEs between carbon fiber and matrix were consistent.

\subsubsection{Between the front and back surfaces along the loading direction}

The distributions of thermal displacements, thermal stresses and thermal strains of the front and back surfaces along the loading direction were also extracted from the nodes between the carbon fiber and matrix and were shown in Fig. 20. The similar phenomena in Fig. 19 can also be seen in Fig. 20, which were no need for more explanations. However, there is other important information conveying from Fig. 20. In the longitudinal direction, the thermal stresses of carbon fiber on the front surface (the loading surface) were negative and on the back surface (parallel to the loading surface) were positive. However, the opposite thermal stress distributions were found in the transverse loading direction. This difference was also attributed to the anisotropic CTEs of carbon fiber. In the longitudinal direction, the negative axial CTE of carbon fiber gave the compressive thermal stress to the front surface and gave the tensile thermal stress to the back surface due to the periodic temperature and displacement boundary conditions. While this trend showed opposite in the transverse direction that the positive radial CTE of carbon fiber gave the tensile thermal stress to the front surface and gave the compressive thermal stress to the back surface. However, the 
thermal stress and thermal strain distributions of the resin-voids mixed matrix between the front and back surfaces in longitudinal loading direction were similar with those in transverse direction. Due to the positive and isotropic CTE of resin-voids mixed matrix, it was suffered small thermal stress and large thermal strain both in the front and back surfaces. And the thermal strain in the front surface was larger than that in the back surface, which was contributed to the loading mode.

\section{Conclusions}

The thermal expansion behaviors of neat cured epoxy resin and carbon fiber/epoxy UD composites were investigated experimentally and numerically. A mesoscale finite element model of UD composites was established based on the periodic temperature and displacement boundary conditions to numerically characterize the thermal expansion behaviors at the temperature range of $25-210^{\circ} \mathrm{C}$. According to the results obtained, the following conclusions could be drawn.

1. The glass transition temperature $T_{g}$ of epoxy resin had a significant effect on the thermal expansion behaviors of neat cured epoxy resin as well as UD composites. The thermal strain and linear CTE curves of epoxy resin appeared an obvious shrinkage during the glassy transition region.

2. The voids could effectively reduce the CTEs of UD composites and composites with higher porosity had lower linear CTEs. Due to the existing voids providing more space for free volume expansion as well as reducing the elastic modulus of resin-voids mixed matrix, the thermal strains and linear CTEs of UD composites could be obviously reduced.

3. Fiber orientation angle also had an obvious effect on the thermal expansion behaviors of UD composite. The bigger was the fiber orientation angle, the higher the linear CTE of UD composites would be.

4. The mesoscale FEA showed better predictive ability of thermal expansion than various analysis models. The CTE of UD composites was lower than that of epoxy resin. For UD composites, the transverse CTE was significantly larger than the longitudinal CTE. The longitudinal CTE was negative after $T_{g}$ and more affected by the axial CTE of carbon fiber, while its transverse CTE was positive and more affected by the CTE of epoxy resin.

5. The study could find that the thermal stresses at the interface were biggest in the longitudinal 
direction and lowest in the transverse direction. In addition, the inconsistent thermal stress state of carbon fiber existed between the front and back surface of the loading direction, and the consistent thermal stress and thermal strain states of resin-voids mixed matrix existed between the front and back surface of the loading direction could be also found.

\section{Acknowledgements}

The authors acknowledge the financial supports from the Chang Jiang Scholars Program and National Science Foundation of China (Grant Number 11272087, 11572085). The financial supports from Foundation for the Fok Ying-Tong Education Foundation (Grant No. 141070), "Shu Guang" project (Grant No. 14SG31) supported by Shanghai Municipal Education Commission and Shanghai Education Development Foundation, Funds for National Engineering and Research Center for Commercial Aircraft Manufacturing (Grant No. SAMC14-JS-15-048), the Fundamental Research Funds for the Central Universities of China, and DHU Distinguished Young Professor Program are also gratefully acknowledged.

\section{References}

[1] Ai S, Fu H, He R, Pei Y. Multi-scale modeling of thermal expansion coefficients of C/C composites at high temperature. Materials \& Design. 2015,82:181-8.

[2] Wang S, Tambraparni M, Qiu J, Tipton J, Dean D. Thermal Expansion of Graphene Composites. Macromolecules. 2009,42(14):5251-5.

[3] Gladysz GM, Chawla KK. Coefficients of thermal expansion of some laminated ceramic composites. Composites Part A Applied Science \& Manufacturing. 2001,32(2):173-8.

[4] Dudescu C, Naumann J, Stockmann M, Nebel S. Characterisation of Thermal Expansion Coefficient of Anisotropic Materials by Electronic Speckle Pattern Interferometry. Strain. 2006,42(3):197-205.

[5] Pan B, Xie HM, Hua T, Asundi A. Measurement of coefficient of thermal expansion of films using digital image correlation method. Polymer Testing. 2009,28(1):75-83.

[6] Esposito M, Buontempo S, Petriccione A, Zarrelli M, Breglio G, Saccomanno A, et al. Fiber Bragg Grating sensors to measure the coefficient of thermal expansion of polymers at cryogenic temperatures. Sensors \& Actuators A Physical. 2013,189(s 3-4):195-203. 
[7] Wang M, Liao Y, Chen D. Determination of linear thermal expansion coefficient of polymeric materials by infrared thermography. Polymer Testing. 2013,32(2):175-8.

[8] Lin J-J, Wu Y-L, Yang C-F, Wang W-W. Measurement of thermal expansion coefficient of INVAR foil using atomic force microscopy. Measurement. 2014,47:373-8.

[9] Lei Z, Wang Y, Qin F, Qiu W, Bai R, Chen X. Multi-fiber strains measured by micro-Raman spectroscopy: Principles and experiments. Optics \& Lasers in Engineering. 2016,77:8-17.

[10] Sauder C, Lamon J, Pailler R. Thermomechanical properties of carbon fibres at high temperatures (up to 2000 C). Composites Science and Technology. 2002,62(4):499-504.

[11] Rupnowski P, Gentz M, Sutter JK, Kumosa M. An evaluation of the elastic properties and thermal expansion coefficients of medium and high modulus graphite fibers. Composites Part A Applied Science \& Manufacturing. 2005,36(3):327-38.

[12] Pradere C, Sauder C. Transverse and longitudinal coefficient of thermal expansion of carbon fibers at high temperatures (300-2500K). Carbon. 2008,46(14):1874-84.

[13] Pradere C, Batsale JC, Goyhénèche JM, Pailler R, Dilhaire S. Thermal properties of carbon fibers at very high temperature. Carbon. 2009,47(3):737-43.

[14] Tezvergil A, Lassila LV, Vallittu PK. The effect of fiber orientation on the thermal expansion coefficients of fiber-reinforced composites. Dental Materials Official Publication of the Academy of Dental Materials. 2003,19(6):471-7.

[15] Zhu RP, Sun CT. Effects of Fiber Orientation and Elastic Constants on Coefficients of Thermal Expansion in Laminates. Mechanics of Advanced Materials \& Structures. 2003,10(2):99-107.

[16] Glavchev I, Petrova K, Ivanova M. Determination of the coefficient of thermal expansion of epoxy composites. Polymer Testing. 2002,21(21):177-9.

[17] Tognana S, Salgueiro W, Somoza A, Pomarico JA, Ranea-Sandoval HF. Influence of the filler content on the thermal expansion behavior of an epoxy matrix particulate composite. Materials Science and Engineering: B. 2009,157(1-3):26-31.

[18] Ghabezloo S. Micromechanical analysis of the effect of porosity on the thermal expansion coefficient of heterogeneous porous materials. International Journal of Rock Mechanics \& Mining Sciences. 2013,55(10):1857-66.

[19] Sideridis E, Kytopoulos VN, Kyriazi E, Bourkas G. Determination of thermal expansion coefficient of particulate composites by the use of a triphase model. Composites Science \& 
Technology. 2005,65(6):909-19.

[20] Chen X-G, Guo J-D, Zheng B, Li Y-Q, Fu S-Y, He G-H. Investigation of thermal expansion of $\mathrm{PI} / \mathrm{SiO} 2$ composite films by $\mathrm{CCD}$ imaging technique from -120 to $200^{\circ} \mathrm{C}$. Composites Science and Technology. 2007,67(14):3006-13.

[21] Kerner EH. The Electrical Conductivity of Composite Media. Proceedings of the Physical Society. 1956,69(8):802-7.

[22] Levin VM. On the coefficients of thermal expansion of heterogeneous materials. Mekhanika Tverdogo Tela 1967:88 [in Russian].

[23] Schapery RA. Thermal expansion coefficients of composite materials based on energy principles. Journal of Composite Materials. 1968,2(3):380-404.

[24] Van Fo Fy GA. Elastic constants and thermal expansion of certain bodies with inhomogeneous regular structure. Soviet Physics, Doklady 1966;11:176 [in Russian].

[25] Van Fo Fy GA. Basic relations of the theory of oriented glass reinforced plastics with hollow fibers. Mekhanika Polimerov 1966;2:763 [in Russian].

[26] Chamis CC, Sendeckyj GP. Critique on Theories Predicting Thermoelastic Properties of Fibrous Composites. Journal of Composite Materials. 1968,2(3):332-58.

[27] Sideridis E. Thermal expansion coefficients of fiber composites defined by the concept of the interphase. Composites Science \& Technology. 1994,51(3):301-17.

[28] Curtu I, Motoc Luca D. Micromechanics of composite materials. Theoretical models. Brasov: Transilvania University Press; 2009

[29] Rosen BW, Hashin Z. Effective Thermal Expansion Coefficients and Specific Heats of Composite Materials. International Journal of Engineering Science. 1970,8(2):157-73.

[30] Sakata S, Ashida F, Kojima T. Stochastic homogenization analysis for thermal expansion coefficients of fiber reinforced composites using the equivalent inclusion method with perturbation-based approach. Computers \& Structures. 2010,88(7-8):458-66.

[31] Tsukrov I, Drach B, Gross TS. Effective stiffness and thermal expansion coefficients of unidirectional composites with fibers surrounded by cylindrically orthotropic matrix layers. International Journal of Engineering Science. 2012,58:129-43.

[32] Lu P. Further studies on Mori-Tanaka models for thermal expansion coefficients of composites. Polymer. 2013,54(6):1691-9. 
[33] Karadeniz ZH, Kumlutas D. A numerical study on the coefficients of thermal expansion of fiber reinforced composite materials. Composite Structures. 2007,78(1):1-10.

[34] Ran Z, Yan Y, Li J, Qi Z, Yang L. Determination of thermal expansion coefficients for unidirectional fiber-reinforced composites. Chinese Journal of Aeronautics. 2014,27(5):1180-7.

[35] Dong C. Development of a Model for Predicting the Transverse Coefficients of Thermal Expansion of Unidirectional Carbon Fibre Reinforced Composites. Applied Composite Materials. 2008,15(3):171-82.

[36] Xu Y, Zhang W. A strain energy model for the prediction of the effective coefficient of thermal expansion of composite materials. Computational Materials Science. 2012,53(1):241-50.

[37] Abueidda DW, Dalaq AS, Abu Al-Rub RK, Jasiuk I. Micromechanical finite element predictions of a reduced coefficient of thermal expansion for 3D periodic architectured interpenetrating phase composites. Composite Structures. 2015,133:85-97.

[38] Wang L, Wang Z, Dong SM, Zhang W, Wang Y. Finite element simulation of stress distribution and development of $\mathrm{Cf} / \mathrm{SiC}$ ceramic-matrix composite coated with single layer $\mathrm{SiC}$ coating during thermal shock. Composites Part B: Engineering. 2013,51:204-14.

[39] Hsueh CH, Becher PF. Thermal Expansion Coefficients of Unidirectional Fiber-Reinforced Ceramics. Journal of the American Ceramic Society. 1988,71(10):438-41.

[40] Motoc DL, Ivens J, Dadirlat N. Coefficient of thermal expansion evolution for cryogenic preconditioned hybrid carbon fiber/glass fiber-reinforced polymeric composite materials. Journal of Thermal Analysis and Calorimetry. 2012,112(3):1245-51.

[41] Zamri MH, Akil HM, Safiee S, Ishak ZAM, Bakar AA. Predicting the Coefficient of Thermal Expansion of Pultruded Composites with a Natural-Fiber Reinforcement. Mechanics of Composite Materials. 2014,50(5):603-12.

[42] Heinle M, Drummer D. Temperature-dependent coefficient of thermal expansion (CTE) of injection molded, short-glass-fiber-reinforced polymers. Polymer Engineering \& Science. 2015,55(11):2661-8.

[43] Pan Z, Gu B, Sun B. Numerical analyses of thermo-mechanical behaviors of 3-D rectangular braided composite under different temperatures. The Journal of The Textile Institute. 2014,106(2):173-86.

[44] Liu C, Yu J, Sun X, Zhang J, He J. Thermal degradation studies of cyclic olefin copolymers. 
Polymer Degradation \& Stability. 2003,81(2):197-205.

[45] Gustafsson SE. Transient plane source techniques for thermal conductivity and thermal diffusivity measurements of solid materials. Review of Scientific Instruments. 1991,62(3):797-804.

[46] He Y. Rapid thermal conductivity measurement with a hot disk sensor. Thermochimica Acta. 2005,436(1-2):122-9.

[47] Nawab Y, Jacquemin F, Casari P, Boyard N, Borjon-Piron Y, Sobotka V. Study of variation of thermal expansion coefficients in carbon/epoxy laminated composite plates. Composites Part B: Engineering. 2013,50:144-9.

[48] Jia X, Xia Z, Gu B. Micro/meso-scale damage analysis of three-dimensional orthogonal woven composites based on sub-repeating unit cells. The Journal of Strain Analysis for Engineering Design. 2012,47(5):313-28.

[49] Needleman A, Tvergaard V. Comparison of Crystal Plasticity and Isotropic Hardening Predictions for Metal-Matrix Composites. Journal of Applied Mechanics. 1993,60(1):70-6.

[50] Sun CT, Vaidya RS. Prediction of composite properties from a representative volume element. Composites Science \& Technology. 1996,56(2):171-9.

[51] Xia Z, Zhang Y, Ellyin F. A unified periodical boundary conditions for representative volume elements of composites and applications. International Journal of Solids and Structures. 2003,40(8):1907-21.

[52] Xia Z, Zhou C, Yong Q, Wang X. On selection of repeated unit cell model and application of unified periodic boundary conditions in micro-mechanical analysis of composites. International Journal of Solids and Structures. 2006,43(2):266-78.

[53] Kuo C-M. Elastic bending behavior of solid orthogonal woven 3-D carbon-carbon composite beams. Composites Science and Technology. 2008,68(3-4):666-72.

[54] Dong K, Zhang J, Jin L, Gu B, Sun B. Multi-scale finite element analyses on the thermal conductive behaviors of 3D braided composites. Composite Structures. 2016,143:9-22.

[55] Hibbit H, Karlsson B, Sorensen E. ABAQUS User Manual, Version 6.12. Simulia, Providence, RI. 2012.

[56] Wang X, Hu Y, Song L, Xing W, Lu H, Lv P, et al. Flame retardancy and thermal degradation mechanism of epoxy resin composites based on a DOPO substituted organophosphorus oligomer. Polymer. 2010,51(11):2435-45. 
[57] Dong K, Gu B, Sun B. Comparisons of thermal conductive behaviors of epoxy resin in unidirectional composite materials. Journal of Thermal Analysis and Calorimetry. 2016,124(2):775-89.

\section{Table 1}

Thermal and mechanical properties of carbon fiber and voids

\begin{tabular}{cccccccccc}
\hline Fiber & $\mathrm{E}_{11} / \mathrm{GPa}$ & $\mathrm{E}_{22}, \mathrm{E}_{33} / \mathrm{GPa}$ & $\mathrm{G}_{12}, \mathrm{G}_{13} / \mathrm{GPa}$ & $\mathrm{G}_{23} / \mathrm{GPa}$ & $v_{12}, v_{13}$ & $v_{23}$ & $\alpha / 10^{-6} \mathrm{C}^{-1}$ & $\rho / \mathrm{kg} \mathrm{m}^{-3}$ \\
\hline T700-12k & 230 & 14 & 9 & 5 & 0.25 & 0.3 & $\frac{\alpha_{1}}{\alpha_{2}, \alpha_{3}}$ & 1800 \\
\hline
\end{tabular}

\begin{tabular}{cccccccc}
\hline Voids & $\mathrm{E} / \mathrm{GPa}$ & $\mathrm{G} / \mathrm{GPa}$ & $v$ & $\mathrm{k} / \mathrm{W} \mathrm{m}{ }^{-1} \mathrm{~K}^{-1}$ & $\mathrm{C}_{\mathrm{p}} / \mathrm{J} \mathrm{kg}^{-1} \mathrm{~K}^{-1}$ & $\alpha / 10^{-6 \circ} \mathrm{C}^{-1}$ & $\rho / \mathrm{kg} \mathrm{m}^{-3}$ \\
\hline Air & $1 \times 10^{-9}$ & $5 \times 10^{-10}$ & $1 \times 10^{-8}$ & 0.025 & 1012 & $3676-4000$ & 1.205 \\
\hline
\end{tabular}

(Notes: the properties of carbon fiber were provided by the supplier, and the properties of voids were from the online material database www.matwed.com.) 


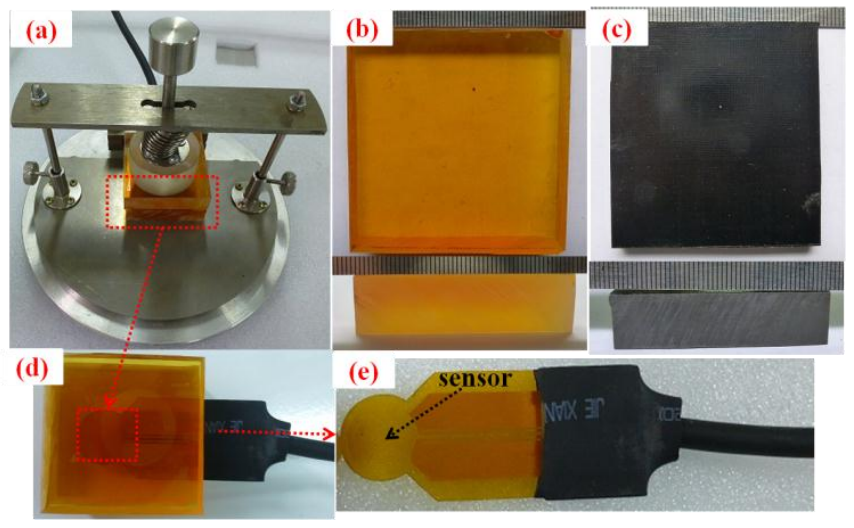

Fig.1. Measurements of the thermal conductivity: (a) the placement of the sample; (b) the neat epoxy resin; (c) the UD composites; (d) the placement of the sensor; (e) the sensor.

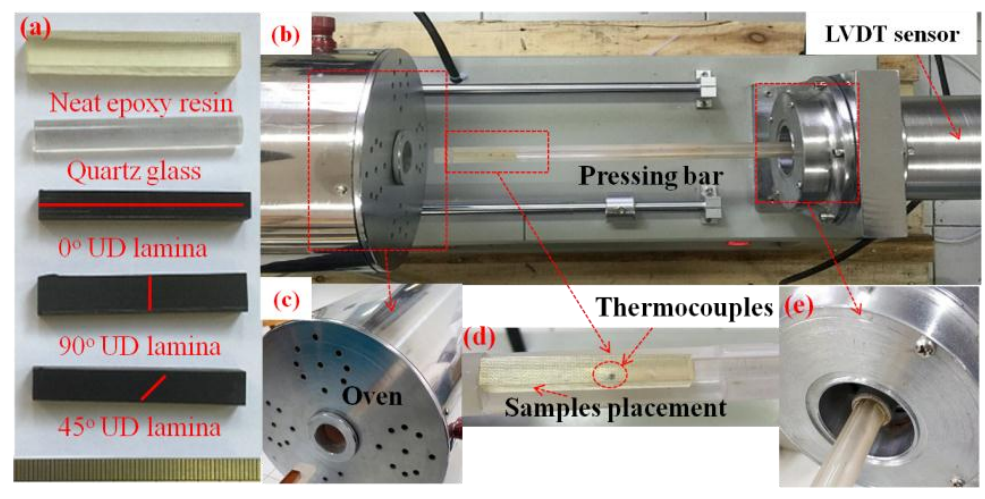

Fig.2. Measurements of the coefficient of thermal expansion: (a) samples for thermal expansion measurements; (b) ZRPY-1000 dilatometer; (c) the heating oven; (d) the placement of the samples; (e) LVDT sensors. (Notes: the red solid lines in (a) represent the fiber orientation directions.) 


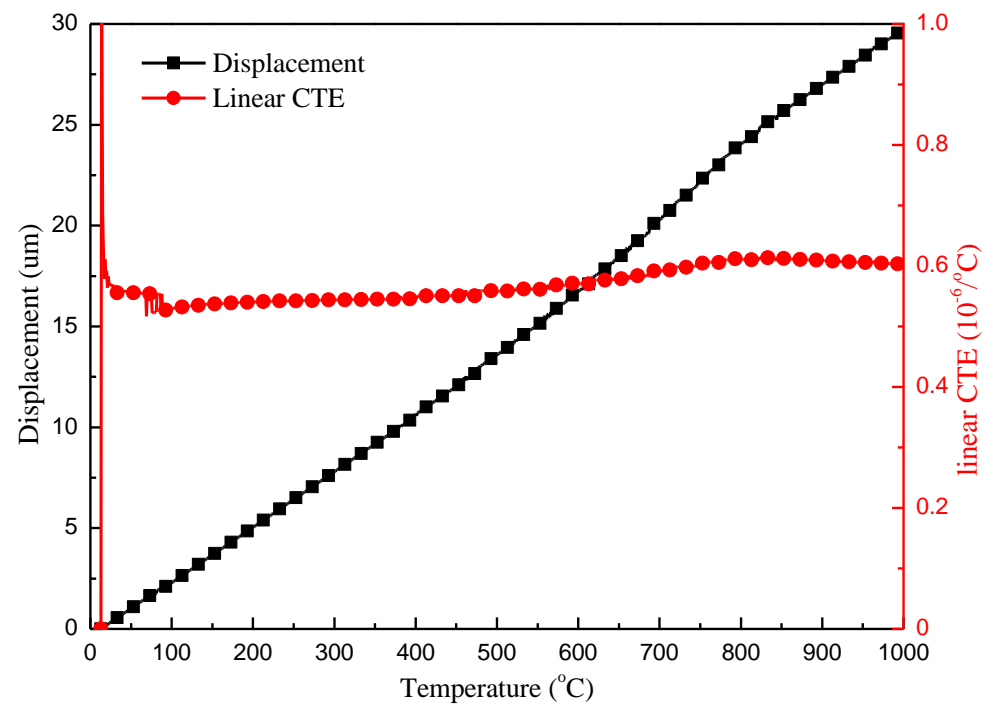

Fig.3. Thermal displacement and linear CTE of the reference quartz glass sample.

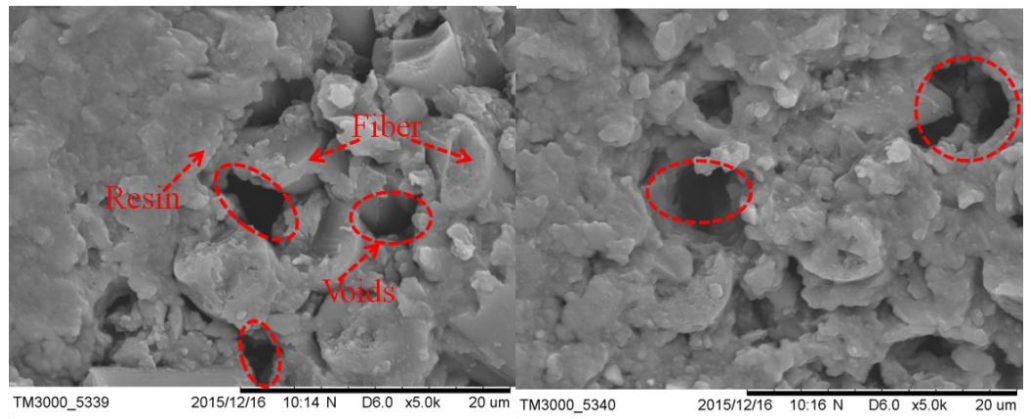

Fig.4. SEM observations of UD composites.

UD composite

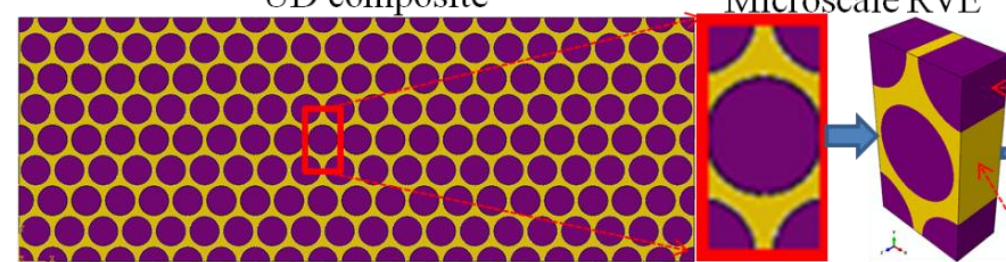

Resin-voids RVE

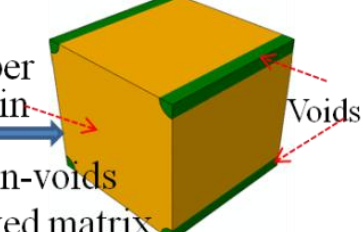

Fig.5. Mesoscale RVE model for UD fiber reinforced composites. 


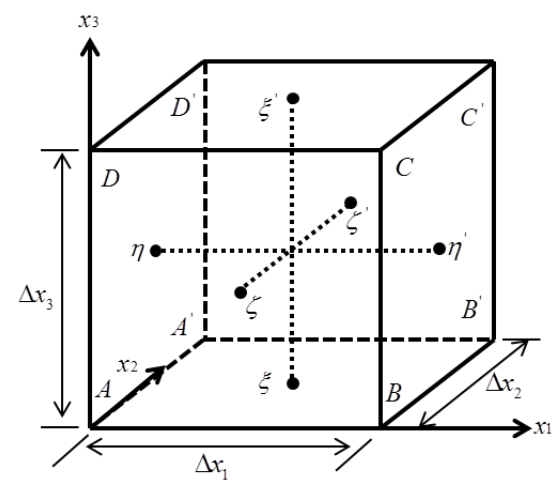

Fig.6. Application of PBCs by master and slave nodes technique.

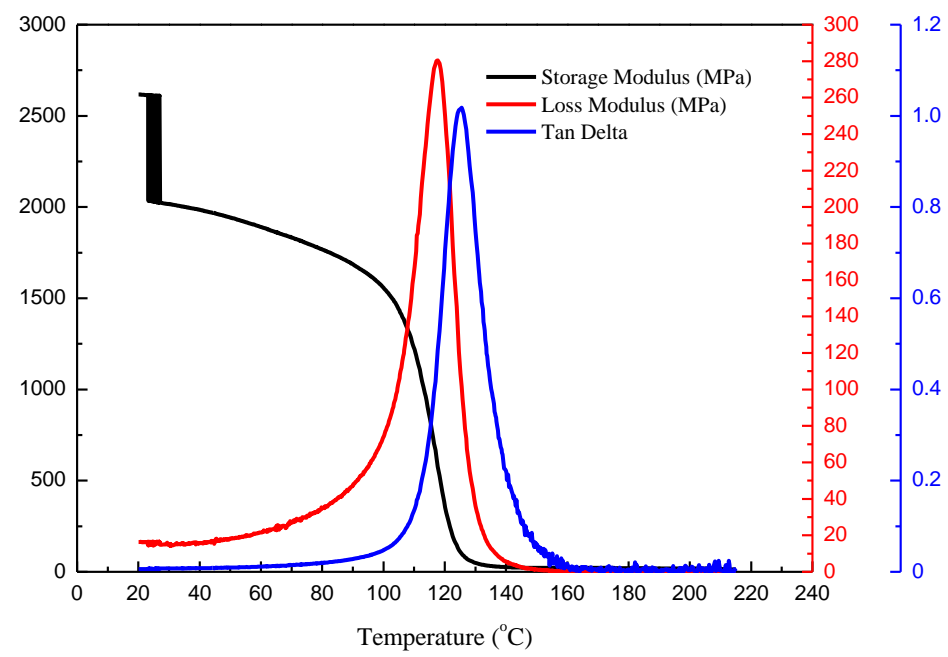

Fig.7. DMA test results of epoxy resin.

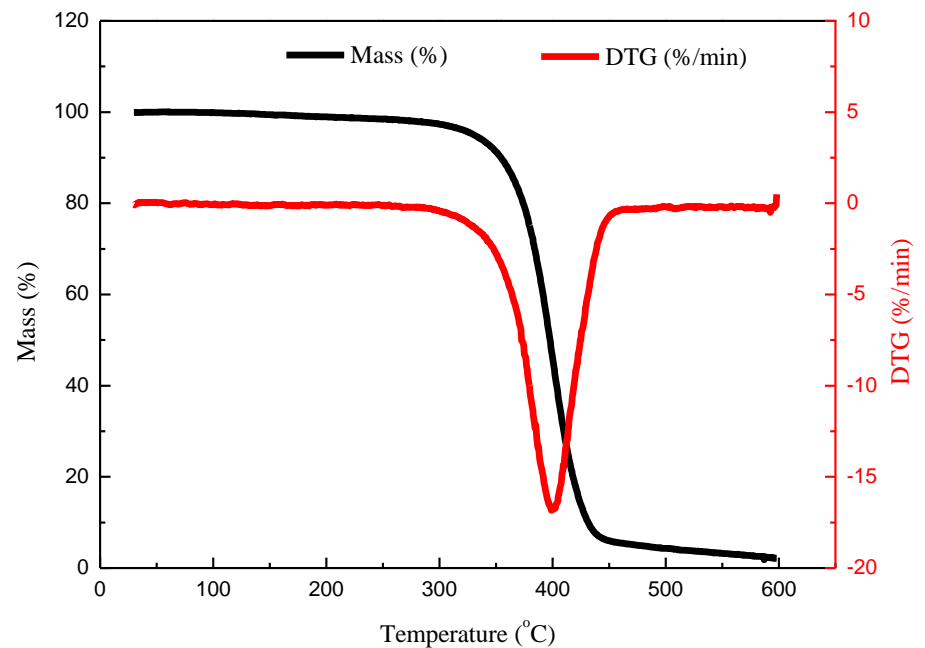

Fig.8. TG test results of epoxy resin. 


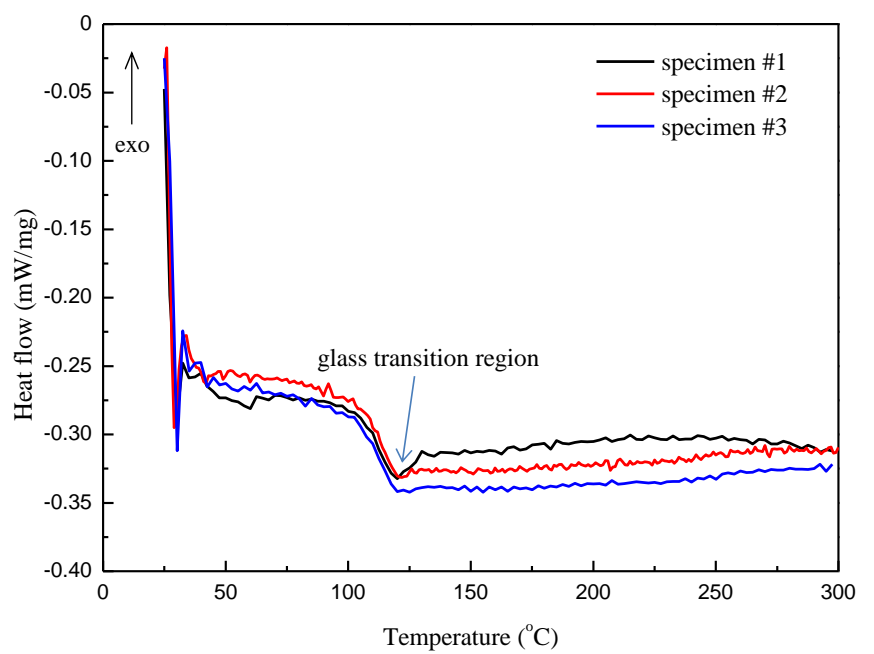

Fig.9. DSC curves for neat epoxy resin.

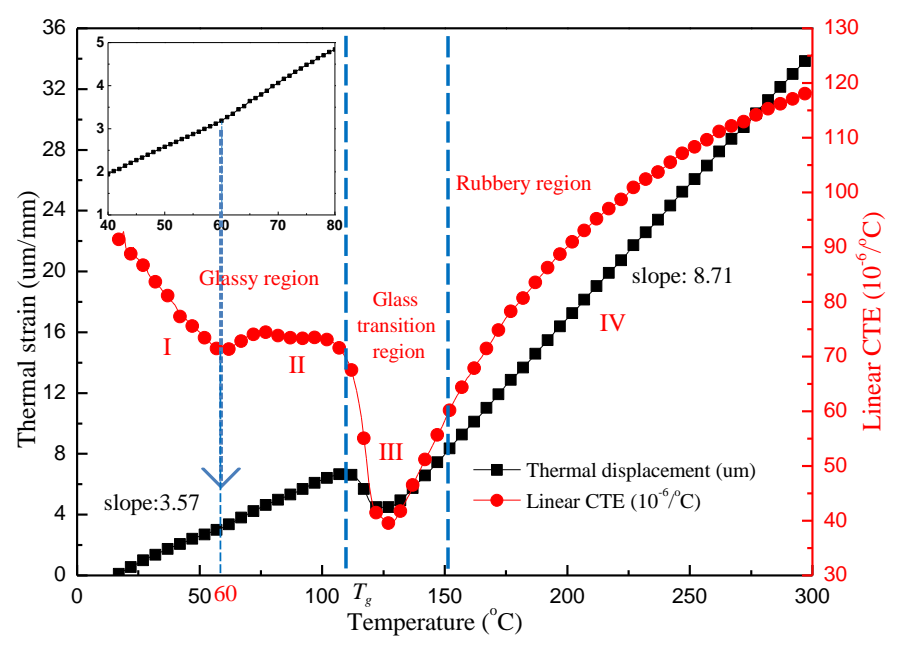

Fig.10. Thermal strains and Linear CTEs as a function of temperature for neat cured epoxy resin. 


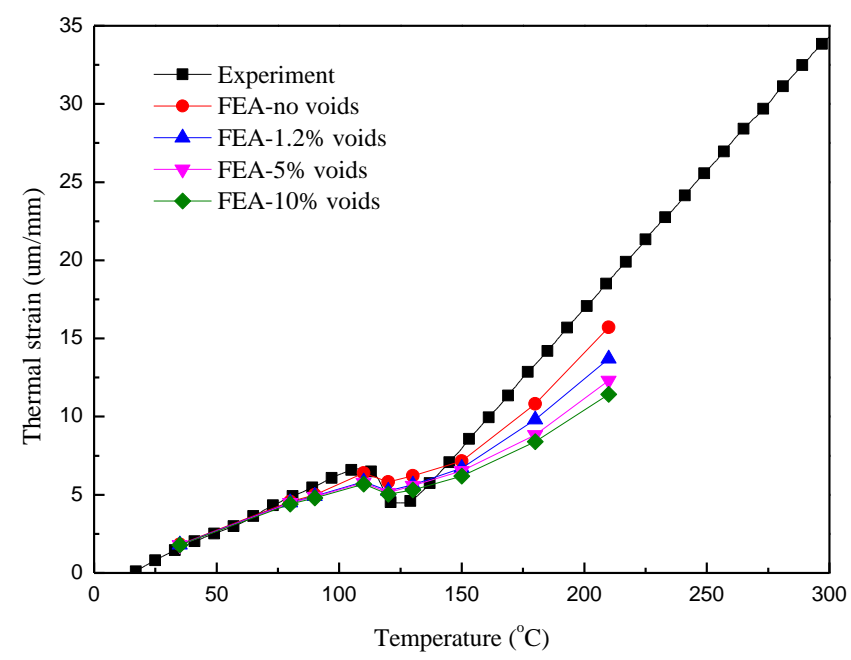

Fig.11. Thermal strains as a function of temperature for resin-voids RVEs with different porosities.

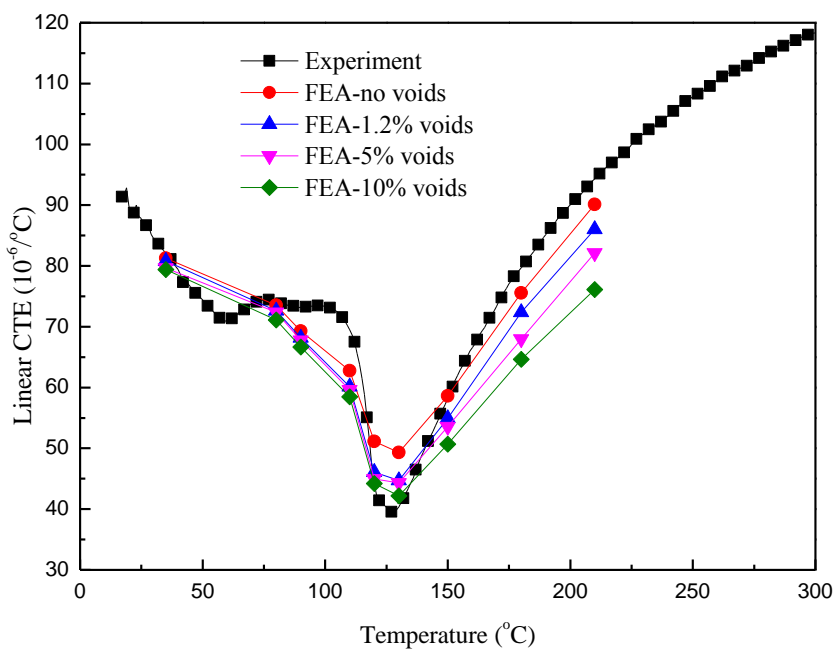

Fig.12. Linear CTEs of resin-voids RVEs with different porosities at different loading temperatures. 


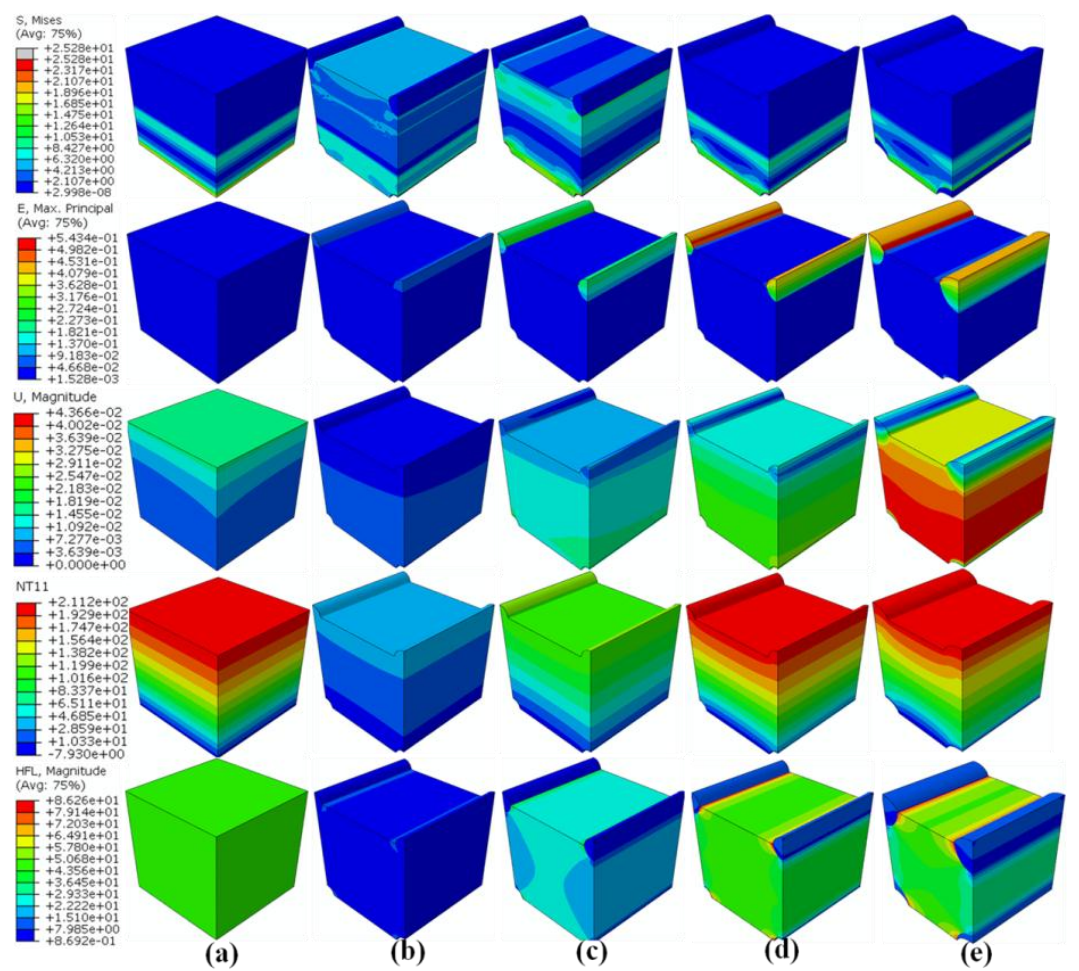

Fig.13. Thermal mechanical properties of resin-voids RVEs with different porosities at different temperature points: (a) $210^{\circ} \mathrm{Cof}$ no voids; (b) $35^{\circ} \mathrm{Cof} 1.2 \%$ voids; (c) $110^{\circ} \mathrm{Cof} 1.2 \%$ voids; (d) $210^{\circ} \mathrm{C}$ of $1.2 \%$ voids; (e) $210^{\circ} \mathrm{Cof} 10 \%$ voids.

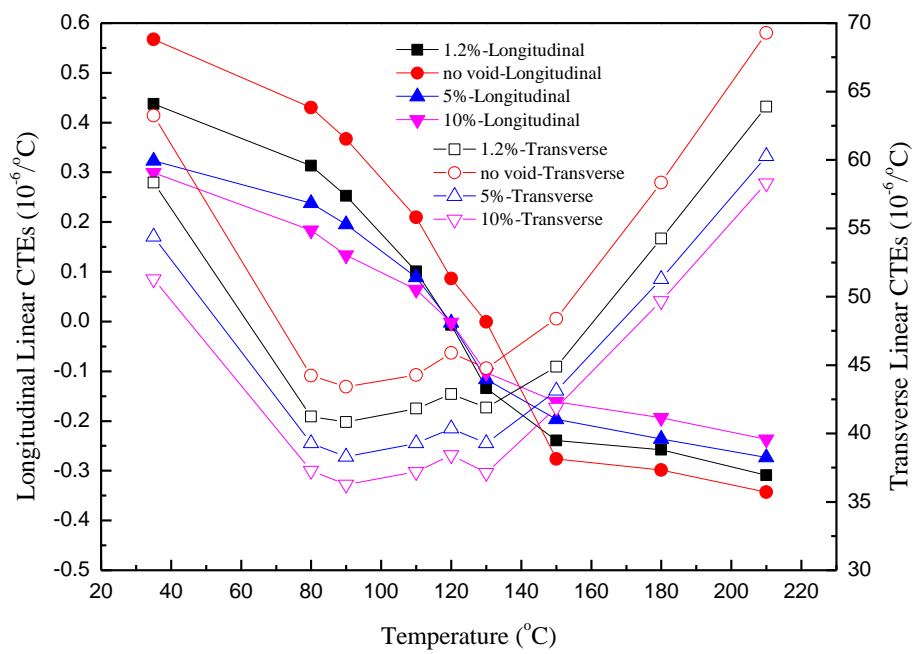

Fig.14. Effect of porosity on the linear CTEs of UD composites. 


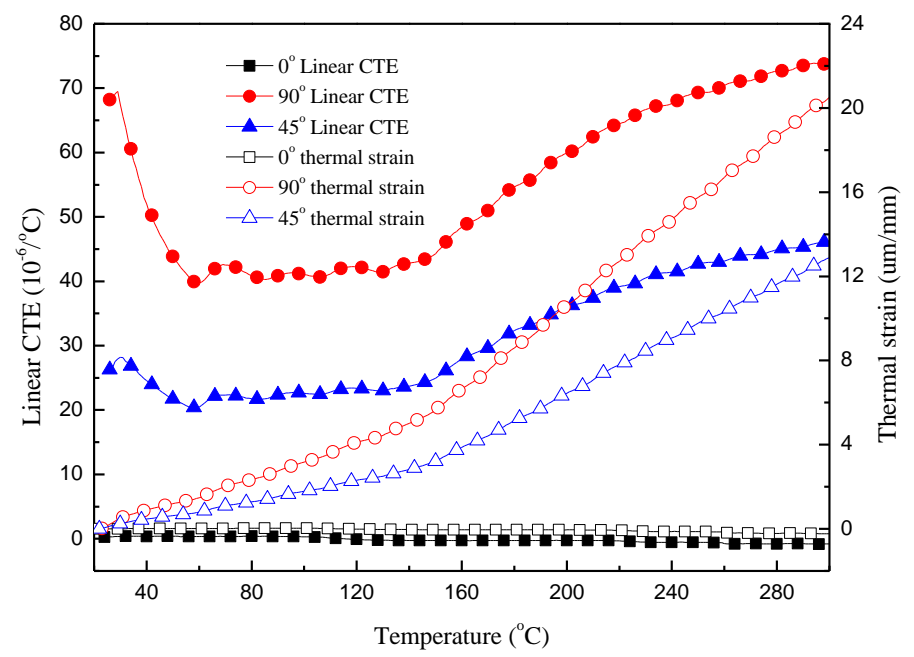

Fig.15. Effect of fiber orientation angle on the thermal expansion properties of UD composites.

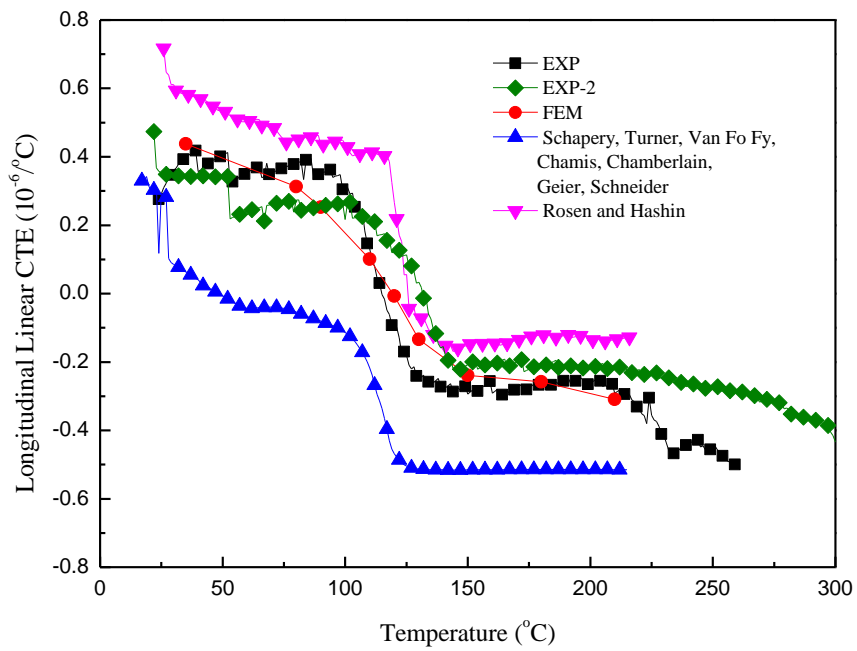

(a) 


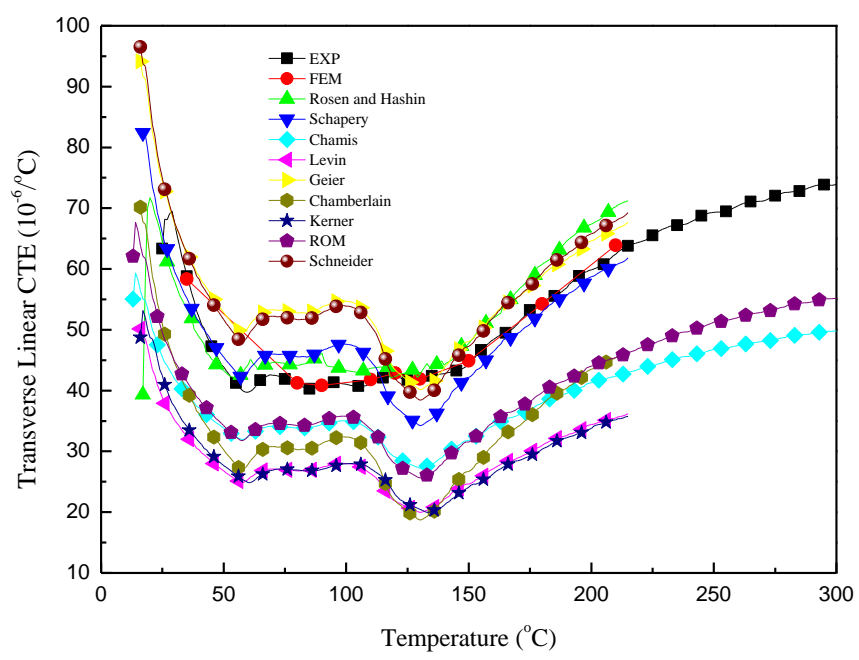

(b)

Fig.16. Comparison of the linear CTE curves as a function of temperature of UD fiber reinforced composites: (a) longitudinal (fiber axial direction) linear CTEs; (b) transverse (fiber radial direction) linear CTEs.

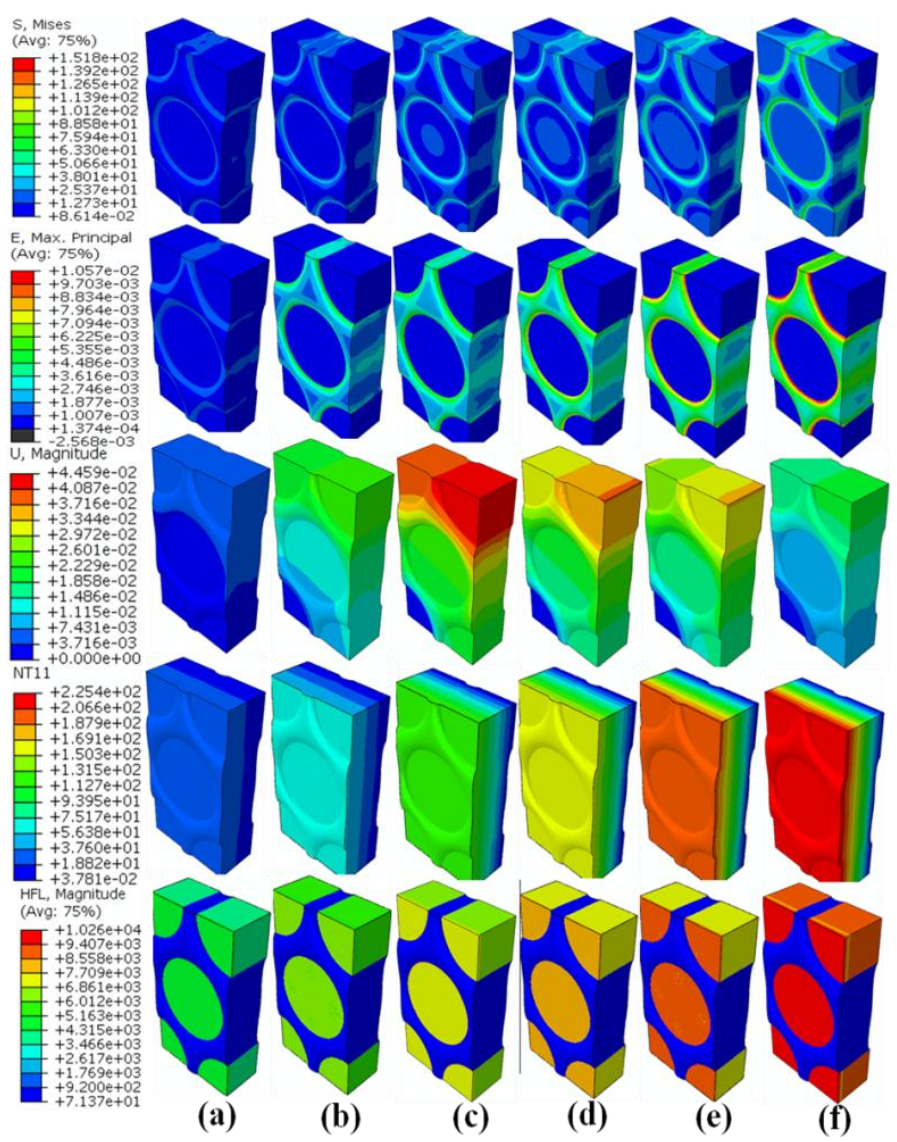

Fig.17.Thermal mechanical behaviors of UD composites in the longitudinal (along fiber axial) 
direction at different loading temperatures: (a) $35^{\circ} \mathrm{C}$; (b) $80^{\circ} \mathrm{C}$; (c) $120^{\circ} \mathrm{C}$; (d) $150^{\circ} \mathrm{C}$; (e) $180^{\circ} \mathrm{C}$; (f) $210^{\circ} \mathrm{C}$.

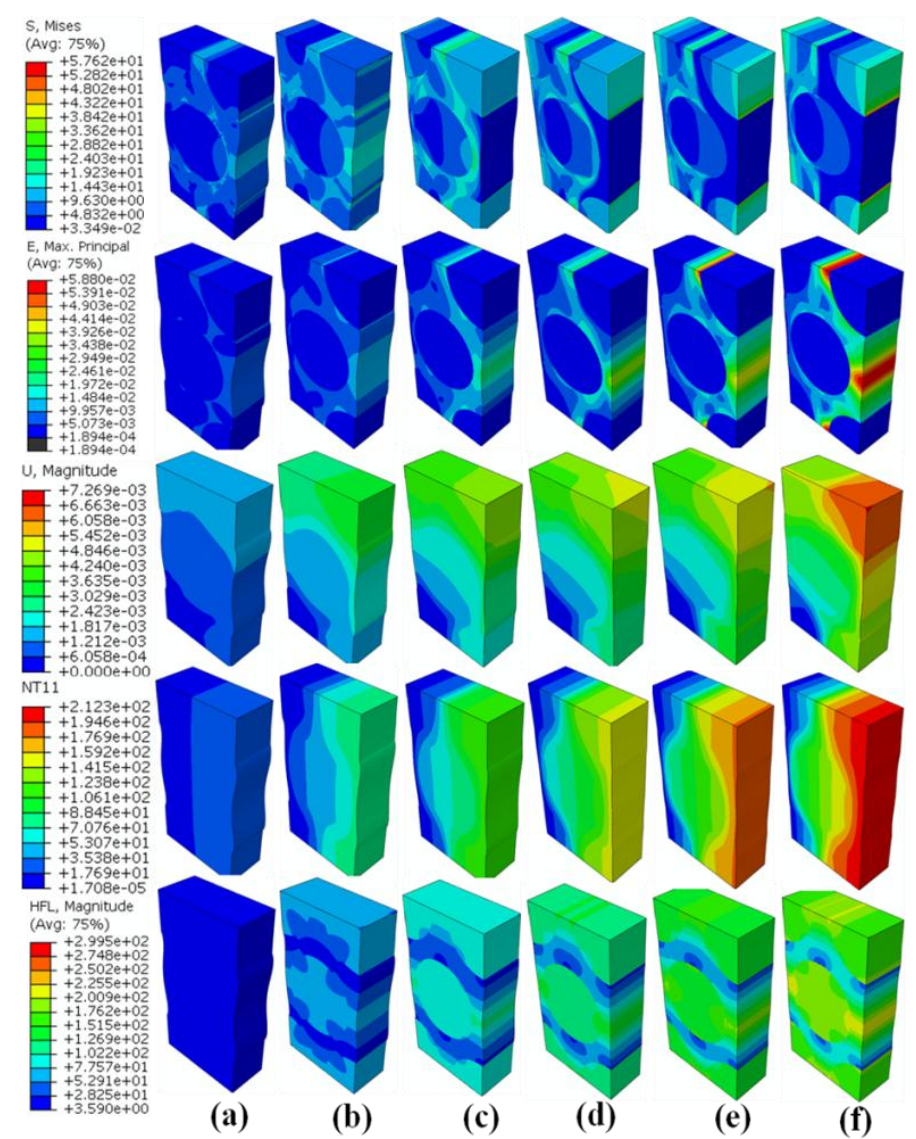

Fig.18. Thermal mechanical behaviors of UD composite in the transverse (along fiber radial) direction at different loading temperatures: (a) $35^{\circ} \mathrm{C}$; (b) $80^{\circ} \mathrm{C}$; (c) $120^{\circ} \mathrm{C}$; (d) $150^{\circ} \mathrm{C}$; (e) $180^{\circ} \mathrm{C}$; (f) $210^{\circ} \mathrm{C}$. 


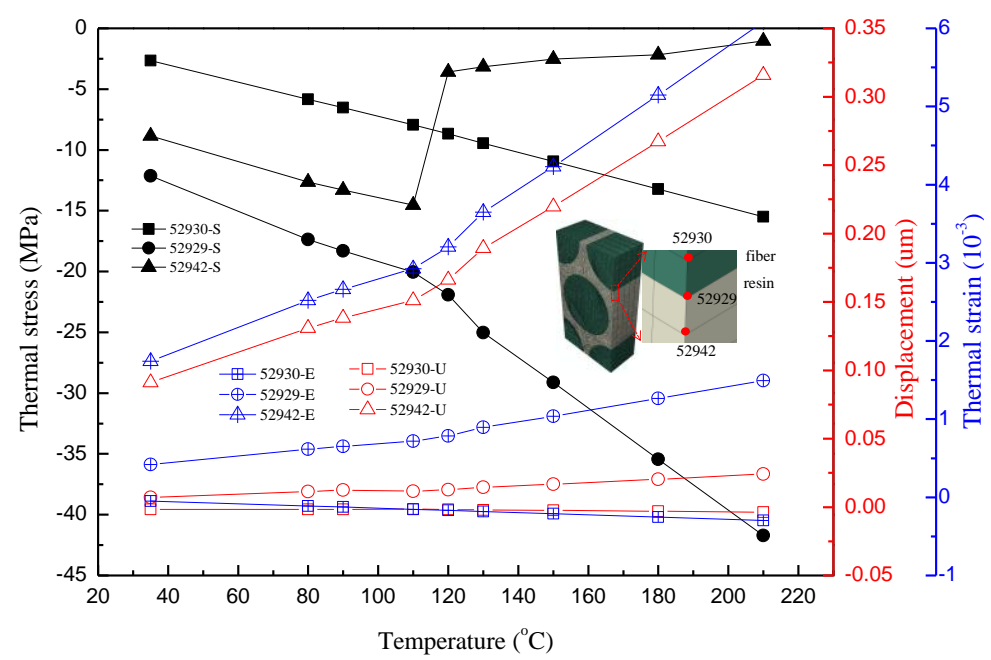

(a)

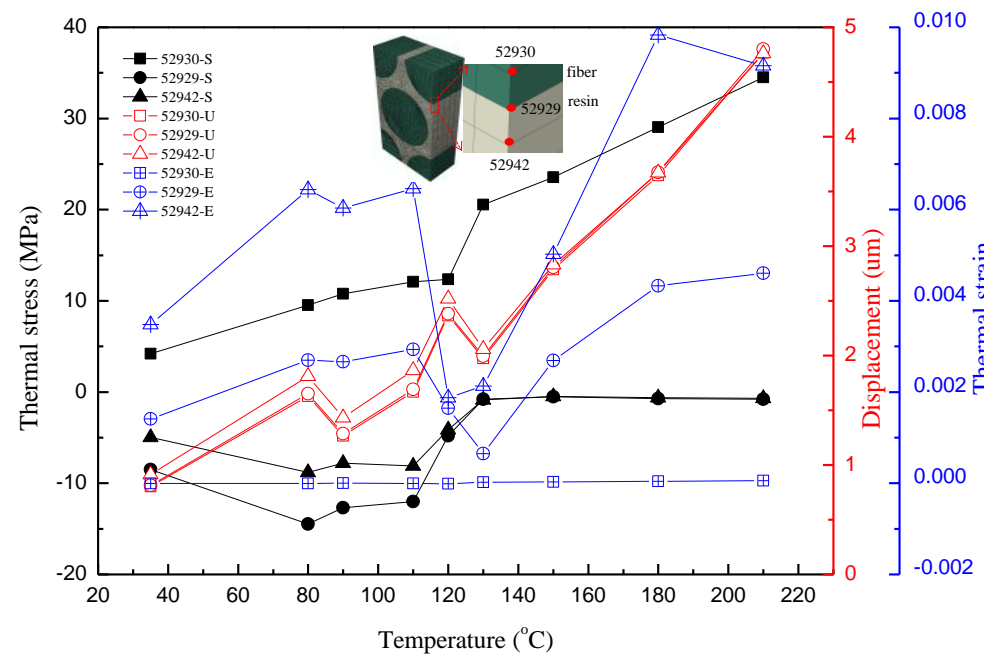

(b)

Fig.19. Distributions of thermal displacement, thermal stress and thermal strain between carbon fiber, resin-voids mixed matrix and their interface: (a) longitudinal direction; (b) transverse direction. (Notes: $\mathrm{S}, \mathrm{U}$ and $\mathrm{E}$ represent the thermal stress, thermal displacement and thermal strain, respectively.) 


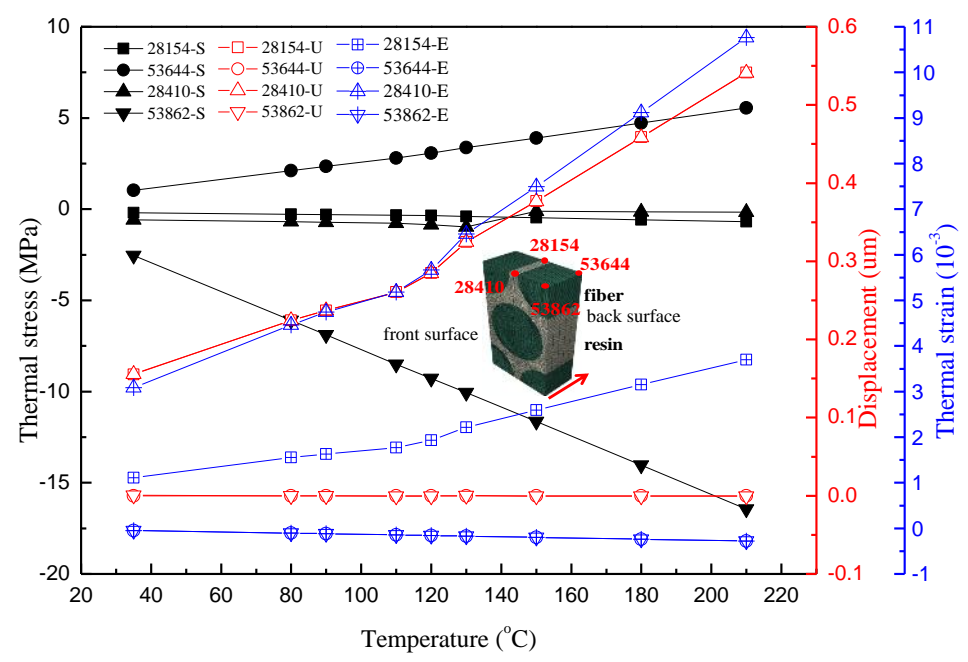

(a)

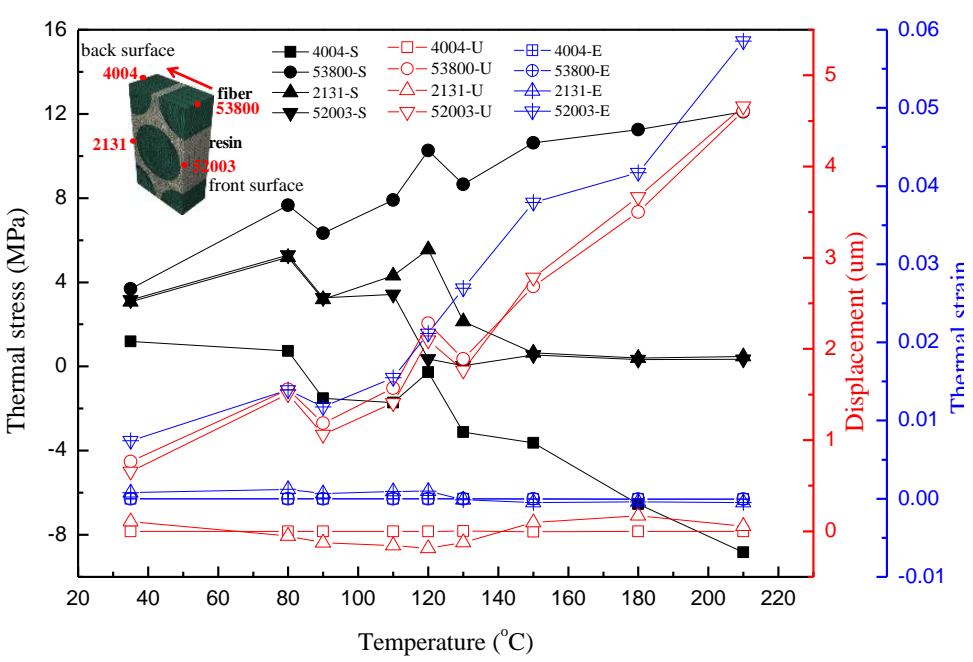

(b)

Fig.20. Distributions of thermal displacement, thermal stress and thermal strain between the front and back surfaces of the loading direction. (Notes: the red arrow directions represent the loading direction of temperature gradient. S, U and E represent the thermal stress, thermal displacement and thermal strain, respectively.) 


\section{Appendix A. Brief description of existing analytical models}

A more comprehensive summary of the existing thermal expansion analytical model has been made in this paper, in order to compare with the current finite element analysis method. A variety of analytical models existing for the prediction of the effective CTEs of UD fiber reinforced composites are raised ranging from the simple mixture law to methods using extremum principles of thermo-elasticity [36]. It is worth noting that the adhesion at the interface between fiber and matrix is assumed to be adequate to withstand the thermal stress in the following models. In addition, in the following equations, ROM, Turner, Kerner, Levin and Schapery were only used for the constituents of isotropic properties, and the longitudinal CTE equation of Chamis was the same as that of Chamberlain, Geier and Schneider.

\section{A.1. Rule of Mixtures (ROM)}

The mixture law is the simplest equation for the CTE evolution. If each phase is assumed homogeneous and isotropic and linearly elastic over a small range of volumetric strains, in the absence of the phase interaction, the CTE of composites may be expected to follow rule of mixtures, just as given by:

$\alpha_{c}=\alpha_{f} V_{f}+\alpha_{m} V_{m}$

where $\alpha_{c}, \alpha_{f}, \alpha_{m}$ are the CTE of composite, carbon fiber and matrix respectively

and $V_{f}$ and $V_{m}$ are the volume fractions of carbon fiber and matrix, respectively.

\section{A.2. Equation of Turner}

In the Turner model, an equal strain approach for the matrix and no restriction on the shape of the fillers is assumed [20]. The equation of CTE is listed as:

$\alpha_{c}=\left(\alpha_{f} E_{f} V_{f}+\alpha_{m} E_{m} V_{m}\right) /\left(E_{f} V_{f}+E_{m} V_{m}\right)$

where $E_{f}$ and $E_{m}$ are the elastic modulus of carbon fiber and matrix.

\section{A.3. Equation of Kerner}

Kerner's model takes into account the effect of shear stresses at interfaces [21]. The Kerner's equation of CTE is listed as: 
$\alpha_{c}=\alpha_{f} V_{f}+\alpha_{m} V_{m}+V_{f} V_{m}\left(\alpha_{f}-\alpha_{m}\right) \frac{E_{f}-E_{m}}{V_{m} E_{m}+V_{f} E_{f}+3 E_{m} E_{f} / 4 G_{m}}$

where $G_{m}$ is the shear modulus of matrix.

\section{A.4. Equation of Levin}

Levin expanded Hill's method and gave the upper bounds of CTE for a certain glass fiber-reinforced composite [22], just as:

$\alpha_{C}=\alpha_{m}-\frac{\left(\alpha_{m}-\alpha_{f}\right) E_{f}\left(3 E_{m}+4 G_{m}\right) V_{f}}{E_{m}\left(3 E_{f}+4 G_{m}\right)+4\left(E_{f}-E_{m}\right) G_{m} V_{f}}$

\section{A.5. Equation of Schapery}

Schapery has derived expressions for longitudinal and transverse CTEs of composites with isotropic fibers embedded in isotropic matrices by adopting extreme energy principles [23]. As a result, for a UD two phase composite, the longitudinal and transverse CTEs are listed as:

$$
\begin{aligned}
& \alpha_{C L}=\left(\alpha_{f} E_{f} V_{f}+\alpha_{m} E_{m} V_{m}\right) /\left(E_{f} V_{f}+E_{m} V_{m}\right) \\
& \alpha_{C T}=\left(1+v_{f}\right) \alpha_{f} V_{f}+\left(1+v_{m}\right) \alpha_{m} V_{m}-\alpha_{C L}\left(v_{f} V_{f}+v_{m} V_{m}\right)
\end{aligned}
$$

where $\alpha_{C L}$ and $\alpha_{C T}$ are the longitudinal and transverse linear CTE of composites, respectively. $v_{f}$ and $v_{m}$ are the Poisson's ratio of isotropic fibers and matrix, respectively.

\section{A.6. Equation of Van Fo Fy}

In a series of articles by Van Fo Fy [24, 25], the CTEs for matrix reinforced by continuous fibers are developed by means of a detailed stress analysis. The CTEs for longitudinal and transverse are listed as:

$$
\begin{aligned}
& \alpha_{C L}=\alpha_{m}-\left(\alpha_{m}-\alpha_{f}\right) \frac{\left(1+v_{m}\right) E_{f} V_{f}-\left(1+v_{f 12}\right)\left(E_{1}-E_{m} V_{m}\right)}{\left(v_{m}-v_{f 12}\right) E_{1}} \\
& \alpha_{C T}=\alpha_{m}+\left(\alpha_{m}-\alpha_{C L}\right) v_{12}-\left(\alpha_{m}-\alpha_{f}\right)\left(1+v_{f 12}\right) \frac{v_{m}-v_{12}}{v_{m}-v_{f 12}}
\end{aligned}
$$

where $v_{f 12}$ and $v_{12}$ are the tangential Poisson's ratio of carbon fiber and composite. $E_{1}$ is the elastic modulus for the longitudinal direction of composite, whose value can be calculated by the simple rule of mixture, as 
$E_{1}=E_{f} V_{f}+E_{m} V_{m}$

\section{A.7. Equation of Chamis}

A simple force balance approach to derive expression of both mechanical and thermal properties of UD composites with transversely isotropic fibers was made by Chamis [26]. The expressions for the longitudinal and transverse CTEs are listed as:

$\alpha_{C L}=\left(\alpha_{f L} E_{f L} V_{f}+\alpha_{m} E_{m} V_{m}\right) /\left(E_{f L} V_{f}+E_{m} V_{m}\right)$

$\alpha_{C T}=\alpha_{f T} \sqrt{V_{f}}+\left(1-\sqrt{V_{f}}\right)\left(1+V_{f} v_{m} E_{f L} / E_{L}\right) \alpha_{m}$

where $\alpha_{f L}$ and $\alpha_{f T}$ are the longitudinal and transverse linear CTEs of carbon fiber respectively. $E_{f L}$ and $E_{f T}$ are the longitudinal and transverse elastic modulus of carbon fiber respectively.

\section{A.8. Equation of Chamberlain}

An alternative model for transverse thermal expansion of UD composites was derived by Chamberlain [27], using plane stress thick walled cylinder equations for the case of transversely isotropic fibers in an isotropic matrix. The expression for CTE in the transverse direction takes the form:

$\alpha_{C T}=\alpha_{m}+\frac{2\left(\alpha_{f T}-\alpha_{m}\right) V_{f}}{\left(V_{m}+F-1\right) v_{m}+\left(V_{m}+F-1\right)\left(1-v_{f T}\right) v_{m} E_{m} / E_{f L}+F+V_{f}}$

where $F$ is a packing factor which accounts for fiber packing geometry, and is equal to 0.9069 for hexagonal packing and for 0.7854 for square packing respectively.

\section{A.9. Equation of Geier}

Geier developed the theoretical model solely for the fiber-reinforced composite materials, either their random or unidirectional distribution [28]. The transverse CTE equation of him is provided as:

$$
\alpha_{C T}=\alpha_{f T} V_{f}+\left(1-V_{f}\right)\left[\alpha_{m}+\left(\alpha_{m}-\alpha_{f L}\right) V_{f} \frac{v_{m}+v_{f} E_{m} / E_{f}}{V_{f}+\left(1-V_{f}\right) E_{m} / E_{f L}}\right]
$$

where $\alpha_{f L}$ and $E_{f L}$ are the longitudinal linear CTE and elastic modulus of carbon fiber 
respectively.

\section{A.10. Equation of Schneider}

Schneider assumed a hexagonal arrangement of cylindrical fiber-matrix elements consisting of a fiber surrounded by a cylindrical matrix jacket [27]. The transverse CTE was derived as the following equation:

$$
\alpha_{C T}=\alpha_{m}-\left(\alpha_{m}-\alpha_{f}\right)\left[\frac{2\left(1+v_{m}\right)\left(v_{m}^{2}-1\right) C}{\frac{1+1.1 v_{f}}{1.1 v_{f}-1}-v_{m}+2 v_{m}^{2} C}-\frac{v_{m} \frac{E_{f}}{E_{m}}}{\frac{1}{C}+\frac{E_{f}}{E_{m}}}\right]
$$

in which

$$
C=\frac{1.1 v_{f}}{1-1.1 v_{f}}
$$

\section{A.11. Equation of Rosen and Hashin}

Rosen and Hashin derived expressions for the effective CTEs of multiphase composites and the summary of their approach for UD fiber reinforced composites with transversely isotropic constituents is given as [29]:

$$
\begin{aligned}
& \alpha_{C L}=\hat{\alpha}_{1}+\left(S_{11}-\hat{S}_{11}\right)\left[\left(\alpha_{f 1}-\alpha_{m 1}\right) P_{11}+\left(\alpha_{f 2}-\alpha_{m 2}\right) 2 P_{12}\right]+\left(S_{12}-\hat{S}_{12}\right)\left[\left(\alpha_{f 1}-\alpha_{m 1}\right) P_{12}+\left(\alpha_{f 2}-\alpha_{m 2}\right) 2\left(P_{22}+P_{23}\right)\right] \\
& \alpha_{C T}=\hat{\alpha}_{2}+\left(S_{12}-\hat{S}_{12}\right)\left[\left(\alpha_{f 1}-\alpha_{m 1}\right) P_{11}+\left(\alpha_{f 2}-\alpha_{m 2}\right) 2 P_{12}\right]+\left(S_{22}-\hat{S}_{22}\right)\left[\left(\alpha_{f 1}-\alpha_{m 1}\right) P_{12}+\left(\alpha_{f 2}-\alpha_{m 2}\right) 2\left(P_{22}+P_{23}\right)\right] \\
& +\left(S_{23}-\hat{S}_{23}\right)\left[\left(\alpha_{f 1}-\alpha_{m 1}\right) P_{12}+\left(\alpha_{f 2}-\alpha_{m 2}\right) 2\left(P_{23}+P_{33}\right)\right]
\end{aligned}
$$

where

$$
\begin{aligned}
& S_{11}=1 / E_{1}, S_{22}=S_{33}=1 / E_{2}, S_{12}=S_{13}=-v_{12} / E_{1}, S_{23}=-v_{23} / E_{2} \\
& P_{11}=\left(A_{22}^{2}-A_{23}^{2}\right) / D e t A, P_{12}=P_{13}=\left(A_{12} A_{23}-A_{12} A_{22}\right) / D e t A, P_{22}=P_{33}=\left(A_{11} A_{22}-A_{12}^{2}\right) / D e t A, P_{23}=\left(A_{12}^{2}-A_{11} A_{23}\right) / D e r A \\
& \text { DetA }=A_{11}\left(A_{22}^{2}-A_{33}^{2}\right)+2 A_{12}\left(A_{12} A_{23}-A_{12} A_{22}\right)
\end{aligned}
$$

In the above equations the terms with and without a hat refer to volume average and effective composite properties respectively. $S_{a b}$ can be written for the effective property, the volume average property and the constituent properties. It is very difficult to find the CTEs of composite using equations of Rosen and Hashin, because the effective mechanical properties, the modulus of the constituents and composite should be firstly determined. 